\title{
Phenolic Compounds as Markers of Wine Quality and Authenticity
}

\author{
Vakarè Merkytė ${ }^{1,2}$, Edoardo Longo $^{1,2, *} \mathbb{C}$, Giulia Windisch ${ }^{1,2}$ and Emanuele Boselli ${ }^{1,2} \mathbb{C}$ \\ 1 Faculty of Science and Technology, Free University of Bozen-Bolzano, Piazza Università 5, \\ 39100 Bozen-Bolzano, Italy; Vakare.Merkyte@natec.unibz.it (V.M.); giulia.windisch@unibz.it (G.W.); \\ emanuele.boselli@unibz.it (E.B.) \\ 2 Oenolab, NOI Techpark South Tyrol, Via A. Volta 13B, 39100 Bozen-Bolzano, Italy \\ * Correspondence: edoardo.longo@unibz.it; Tel.: +39-0471-017691
}

Received: 29 October 2020; Accepted: 28 November 2020; Published: 1 December 2020

\begin{abstract}
Targeted and untargeted determinations are being currently applied to different classes of natural phenolics to develop an integrated approach aimed at ensuring compliance to regulatory prescriptions related to specific quality parameters of wine production. The regulations are particularly severe for wine and include various aspects of the viticulture practices and winemaking techniques. Nevertheless, the use of phenolic profiles for quality control is still fragmented and incomplete, even if they are a promising tool for quality evaluation. Only a few methods have been already validated and widely applied, and an integrated approach is in fact still missing because of the complex dependence of the chemical profile of wine on many viticultural and enological factors, which have not been clarified yet. For example, there is a lack of studies about the phenolic composition in relation to the wine authenticity of white and especially rosé wines. This review is a bibliographic account on the approaches based on phenolic species that have been developed for the evaluation of wine quality and frauds, from the grape varieties (of $V$. vinifera and non vinifera), to the geographical origin, the vintage year, the winemaking process, and wine aging. Future perspectives on the role of phenolic compounds in different wine quality aspects, which should be still exploited, are also outlined.
\end{abstract}

Keywords: phenolic compounds; chemical markers; wine authenticity; wine quality; food traceability; chemometrics

\section{Introduction}

Wine is a product with high commercial value and relevant cultural aspects. Its desirability on the market, combined with the high prices that consumers are willing to pay for top quality bottles, is a cause for food frauds [1,2], also with very recent examples [3]. Recently, over one million liters of counterfeit wine were discovered by the European Anti-Fraud Office [4]. Mislabeling of variety, geographical origin, or vintage year and adulteration with ethanol, sugar, and colorants are typical examples of frauds related to wine [5]. Therefore, the wine industry and consumers are highly concerned about the quality and authenticity of wine [6].

Wine quality is determined by several factors such as the type (or blend) of grape varieties, the terroir, the viticultural practices, the winemaking techniques, and the aging conditions [7-9]. The variety of grapes is a key factor in determining the wine flavor, especially during the production of premium wines. Thus, the adulteration of these types of wines with cheaper grape varieties is common [10-12]. Terroir is a French term that defines the very specific combination of geographical, climatic, and pedological factors, characterizing the growth and quality of the grapes. Terroir is mainly influenced by the climate and soil conditions, and it is strongly related to viticultural practices and vintage year [13-15]. 
One of the aspects related to wine authenticity is based on the (blend of) grape varieties used in winemaking, their geographical origin, and vintage. The authenticity related to the terroir is guaranteed by strict guidelines adopted by the European Union also based on national rules and the indications of The International Organization of Vine and Wine [16].

Wine quality evaluation is based on sensory and chemical analyses. In the sensory tasting, wine quality indicators, such as color, mouthfeel, and taste are largely, but not exclusively, influenced by the phenolic profile. Thus, phenolic compounds are widely used for the wine quality and authenticity assessment [17-19].

The aim of this paper is to present an overview of the current knowledge on the phenolic compounds used as chemical markers for specific winemaking practices and the assessment of wine quality and authenticity. In contrast with recent reviews on phenolic markers, this review is focusing on chemical compounds rather than analytical techniques that have been applied and statistical approaches used to process the analytical data [20,21]. The main reported aspects are related to the grape varieties (of $V$. vinifera and non vinifera), the geographical origin, the vintage year, the winemaking process, and wine aging.

\section{Classification of Phenolic Compounds of Wine}

Phenolic compounds are secondary metabolites present in grapes and wine that can be formed and transformed during the winemaking process. Phenolics can be classified as flavonoids (e.g., anthocyanins, flavan-3-ols, flavonols) and non-flavonoids (e.g., phenolic acids, stilbenes) [22,23]. The phenolic compounds most usually applied for the quality and authenticity assessment of wine are phenolic acids, flavonoids, tannins, and stilbenes. The role of each class of phenolics will be discussed.

\subsection{Phenolic Acids}

There are two main groups of phenolic acids that are used for the quality and authenticity assessment and are significant for white grapes and wines: hydroxybenzoic acids (containing seven carbon atoms) and hydroxycinnamic acids (nine carbon atoms). Model structures are reported in Figure 1.<smiles></smiles>

Figure 1. Model structures for common natural hydroxybenzoic acids, cinnamic acids, and an example of a derivative present in grape and in wine. Hydroxylation substitutions on aromatic rings are indicatively shown by the curled bonds.

Cinnamic acids (e.g., caffeic, coumaric, ferulic, and sinapic) can be found also in two isomeric forms (cis and trans) because of the presence of a double bond. Hydroxybenzoic and hydroxycinnamic acids do not only occur in their free forms but as derivatives in conjugated or esterified forms as well. For example, hydroxycinnamic acids in wine originate during fermentation from the hydrolysis of 
hydroxycinnamic tartaric esters $[13,24]$. They can be an oxidation substrate and precursors of browning of white wines and give a bitter flavor [13,25].

\subsection{Flavonoids}

Flavonoids are 15-carbon compounds including two aromatic rings bound through a three-carbon chain. Model general structures for the most common families of natural flavonoids are reported in Figure 2.<smiles>OC1Cc2ccccc2OC1c1ccccc1</smiles><smiles>O=c1cc(-c2ccccc2)oc2ccccc12</smiles>
anthocyanidins<smiles>Oc1cc2ccccc2[o+]c1-c1ccccc1</smiles><smiles>O=c1c(O)c(-c2ccccc2)oc2ccccc12</smiles><smiles>O=C1c2ccccc2OC(c2ccccc2)C1O</smiles>

flavonols

dihydroflavonols (flavanonols)

Figure 2. Model structures for common natural flavonoids. Hydroxylation substitutions and stereogenic configuration patterns are not explicitly shown for brevity.

Wine flavonoids occur both in free and conjugated forms, as for example, glucosides. The most important classes of flavonoids that have been applied as chemical markers are anthocyanins, flavonols, and flavan-3-ols. The most common mono-glycosylated anthocyanin forms are summarized in Figure 3. Anthocyanins can be classified into mono-, disubstituted and trisubstituted congeners according to the total number of hydroxyl and methoxy groups present in the lateral ring (they can be 2 or 3 considering $R^{1}, R^{2}$, and $R^{3}$ in Figure 3). Anthocyanins are not only found in simple mono-glycosylated forms, but they can also be esterified on the glycosidic moiety, such as acetyl-glucosides, $p$-coumaroyl-glucosides, and caffeoyl-glucosides (acylated anthocyanins).

\begin{tabular}{|c|c|c|c|c|c|}
\hline & Common name & Species & $\mathbf{R}^{1}$ & $\mathbf{R}^{2}$ & $\mathbf{R}^{3}$ \\
\hline & Oenin & Malvidin-30-glucoside & $\mathrm{OCH}_{3}$ & $\mathrm{OH}$ & $\mathrm{OCH}_{3}$ \\
\hline & Myrtillin & Delphinidin-3O-glucoside & $\mathrm{OH}$ & $\mathrm{OH}$ & $\mathrm{OH}$ \\
\hline & Oxycoccicyanin & Peonidin-30-glucoside & $\mathrm{H}$ & $\mathrm{OH}$ & $\mathrm{OCH}_{3}$ \\
\hline & $\begin{array}{l}\text { Kuromanine } \\
\text { Chrysontemin } \\
\text { Glucocyanidin }\end{array}$ & Cyanidin-30-glucoside & $\mathrm{H}$ & $\mathrm{OH}$ & $\mathrm{OH}$ \\
\hline & Callistephin & Pelargonidin-30-glucoside & $\mathrm{H}$ & $\mathrm{OH}$ & $\mathrm{H}$ \\
\hline $\mathrm{OH}$ & Not found & Petunidin-30-glucoside & $\mathrm{OCH}_{3}$ & $\mathrm{OH}$ & $\mathrm{OH}$ \\
\hline
\end{tabular}

Figure 3. Structures of mono-glycosylated anthocyanins.

Each grape variety presents a typical anthocyanin pattern [26,27]; thus, anthocyanins are the most applied flavonoids for the assessment of authenticity and quality of red wines. These protocols are based on the differentiation between (a) different anthocyanidin congeners, (b) anthocyanins monoand di-glucosides (3-O-glucoside derivatives are shown in Figure 3), and (c) acylated or non-acylated anthocyanins, as reported in the section Grape variety of red wine. 
Flavonols show an unsaturation between $C_{2}$ and $C_{3}$. They are hydroxylated in position $C_{3}$ and have a carbonyl group in $\mathrm{C}_{4}$. Flavonols are present in wine as aglycones and glycosylated forms [28]. The most abundant flavonols in wines are quercetin and myricetin [29]. Differently from flavonols, flavan-3-ols have a saturated carbon bond between $C_{2}$ and $C_{3}$ and no carbonyl group. Catechin, epicatechin, and their derivatives (e.g., gallocatechin, epicatechin gallate) are the most abundant flavan-3-ols in wine. Some derivatives show also the presence of an ester with gallic acid at $C_{3}$ [30]. A particular type of flavonoids, condensed tannins (or proanthocyanidins), are oligomeric or polymeric forms of flavan-3-ols and will be discussed separately.

\subsection{Tannins}

Tannins are divided into two very different chemical classes: hydrolyzable and condensed tannins; they give the astringency perception to wines [31]. Depending in which acid they are converted upon hydrolysis, hydrolysable tannins are defined as gallotannins (hydrolyzing into gallic acid) or ellagitannins (hydrolyzing into ellagic acid) [32].

Unlike hydrolysable tannins, condensed tannins can be oligomers or polymers of flavan-3-ols, depending on their degree of polymerization. Condensed tannins are also called proanthocyanidins and their degree of polymerization (DP) may range from 2 to about 20 in wine, and their solubility tends to decrease with the increasing number of monomeric units [33,34]. They show a great variability of isomers, depending on the geometry of their bindings and the type of monomers involved (Figure 4). They influence the taste (bitterness), astringencyin wine [35] and color stabilization in red wines by combining with anthocyanins [36].<smiles>Oc1cc(O)c2c(c1)O[C@H](c1ccc(O)c(O)c1)[C@H](O)[C@H]2c1c(O)cc(O)c2c1O[C@H](c1ccc(O)c(O)c1)C(O)C2</smiles><smiles>[B]c1ccc([C@H]2Oc3c(O)cc(O)c(C4c5c(O)cc(O)cc5O[C@@H](c5ccc(O)c(O)c5)C4O)c3O[C@H]2O)cc1O</smiles><smiles>Oc1cc(O)c2c(c1)O[C@H](c1ccc(O)c(O)c1)[C@H](O)[C@H]2c1c(O)cc(O)c2c1O[C@H](c1ccc(O)c(O)c1)C(O)C2</smiles><smiles>Oc1cc(O)c2c(c1)O[C@H](c1ccc(O)c(O)c1)[C@H](O)[C@H]2c1c(O)cc(O)c2c1O[C@H](c1ccc(O)c(O)c1)[C@H](O)C2</smiles>

Figure 4. Example of isomerism for dimeric procyanidins: procyanidin B1 (A), procyanidin B2 (B), procyanidin B3 $(\mathbf{C})$, procyanidin B4 (D).

\subsection{Stilbenes}

The main stilbenes present in grapes and wine are resveratrol and piceid (resveratrol glucosides) in cis and trans isomeric forms (Figure 5). Stilbenes have proved to be good discriminants of the grape variety $[10,23,37-39]$, grape species [5] and terroir $[7,8,13,40]$ in white, rosé, and red wines. 

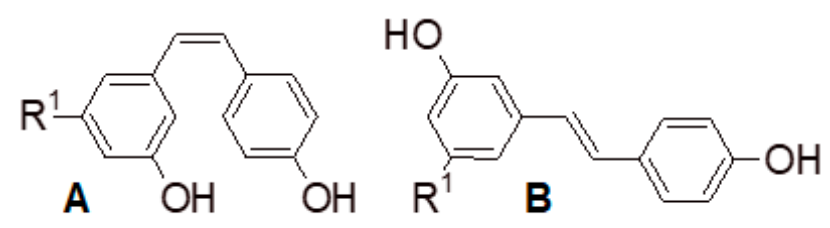

Figure 5. cis-piceid (A) and trans-piceid (B), when $\mathrm{R}^{1}=\mathrm{O}$-glucose; and cis-resveratrol (A) and trans-resveratrol (B), when $\mathrm{R}^{1}=\mathrm{OH}$.

\section{Applications}

\subsection{Grape Variety}

\subsubsection{Grape Variety of White and Rosè Wine}

The comparison of phenolic profiles of different grape cultivars is the most studied application regarding phenolics as chemical markers and is approached using combined analytical techniques such as liquid chromatography, mass spectrometry, nuclear magnetic resonance (NMR), ultraviolet-visible spectroscopy (UV-Vis), and vibrational spectroscopy [41]. Hydroxycinnamic and hydroxybenzoic acids play an important role in the differentiation of wines made from white grapes, as it was shown (Table 1) in the studies on Czech wines [42] and on Romanian and French wines [43]. The most abundant phenolic acids in wines made from local Czech grapes were protocatechuic acid (Aurelius), gallic acid (Moravian Muscat), hydroxybenzoic acids and caftaric acid (Malverina), and $p$-coutaric and caftaric acids (Hibernal). However, the differences were not significant and could have been affected by the winemaking technology. Lampir et al. [42] applied canonical variate analysis (CVA) and showed that hydroxycinnamic acids, (+)-catechin and (-)-epicatechin were the best discriminants and were able to classify all varietal white wines $100 \%$ correctly. Magdas et al. [43] used the content of hydroxycinnamic acids in a combination with shikimic acid and (-)-epicatechin (measured by NMR) for the classification of Chardonnay, Riesling, and Sauvignon Blanc. The results using these particular groups of markers were not affected by the different geographical origins (various regions of France and Romania). Other differentiations of white wines were based on the specific set of phenolics from different classes [10] and in a combination of amino and organic acids [44], shikimic acid, caftaric acid, 2,3-butanediol, glycerol and ethanol [45], 2-S-glutathionylcaftaric acid, and sensory perception [46].

A major study on white, rosé, and red Apulian wines and their blends has been done by Ragusa et al. [23]. Most rosé wines were composed of Negroamaro grapes and yielded similar concentrations of gallic and syringic acids. All rosé wines showed higher values of trans-resveratrol in comparison with white wines. Baron et al. [37] focused on the phenolic components of 48 rosé wines from the Czech Republic. Anthocyanins and caftaric acid were the most abundant phenolic compounds in different grape varieties. Wines made from Blaufränkisch grapes were rich of hydroxycinnamic acids, while Pinot Noir and Zweigeltrebe wines had distinctive profiles (very high and low concentrations, respectively) of catechin, epicatechin, and both isomeric forms of resveratrol. In both [23,37], a set of various phenolics was selected for the possible discrimination of these wines.

\subsubsection{Grape Variety of Red Wine}

For the quality control of red grape varieties, the profile of anthocyanins is typically used. These compounds are presented in Table 2. Red grape cultivars of Vitis vinifera contain a specific profile (relative proportions) of (mostly) monoglucosylated anthocyanins, whereas other Vitis species (so-called American varieties, not admitted to produce wine marketed in the EU) contain also diglucosylated anthocyanins, which are discussed in the next chapter.

Cejudo-Bastante et al. [22] have analyzed Tempranillo, Tortosi, Bobal, Moravia Agria, Moravia Dulce, and Rojal wines. The observed anthocyanins were free, acetyl-, coumaryl, and caffeoyl-esters of cyanidin-3-O-glucoside, delphinidin-3-O-glucoside, malvidin-3-O-glucoside, peonidin-3-O-glucoside, 
and petunidin-3-O-glucoside. In Tempranillo wines, the concentrations of 3-O-glucosides were distributed as follows:

(1) glucosides: Mv-3-glu > Pt-3-glu $>$ Dp-3-glu $>$ Pn-3-glu > Cy-3-glu;

(2) 3-acetyl-glucosides: Mv-3-ace $>$ Pt-3-ace $>$ Pn-3-ace $>$ Dp-3-ace $>$ Cy-3-ace;

(3) 3-p-coumaryl-glucosides: trans-Mv-3-cum > Dp-3-cum > Pn-3-cum > cis-Mv-3-cum > Cy-3-cum;

(4) caffeoyl-3-glucosides: $\mathrm{Mv}$-caf $>$ Pn-caf. $(\mathrm{Mv}=$ malvidin; $\mathrm{Pt}=$ petunidin; $\mathrm{Dp}=$ delphinidin; $\mathrm{Pn}=$ peonidin $; \mathrm{Cy}=$ cyanidin)

Pt-3-cum was not present. In comparison to Tempranillo wines, the concentration of Mv-3-glu in Tortosi wines was twice lower and Cy-3-glu, Pt-3-ace, Pn-3-ace, Dp-3-cum, and Pn-caf were not present. Conversely, Bobal wines had rather low concentrations of all anthocyanins, but only Pt-3-ace, Pn-3-ace, and Pn-caf were not identified. The only anthocyanins that were present in Moravia Agria wines were Cy-3-ace, Mv-3-ace, cis-Mv-3-cum, Pn-3-cum, trans-Mv-3-cum, and Mv-caf; in Moravia Dulce: Dp-3-glu, Pt-3-glu, Pn-3-glu, Mv-3-glu, Cy-3-ace, Mv-3-ace, cis-Mv-3-cum, Pn-3-cum, and trans-Mv-3-cum; in Rojal: Pn-3-glu, Mv-3-glu, and Cy-3-ace.

The absence of a specific anthocyanin in grapes can be exploited for the authenticity assessment. La Notte et al. [47] and Storchi et al. [48] noticed that acetylated anthocyanins and Mv-caf were not present or present in traces in Sangiovese grapes and wines. Instead, these grapes had very low concentrations of 3-p-coumaryl-glucosides that were very abundant in Malbec and Syrah grapes.

Pinot Noir, Pinot Meunier, and Pinot Madeleine grapes do not synthesize acetylated anthocyanins, as well [49].

Revilla et al. [27] have studied anthocyanins in Cabernet Sauvignon, Tempranillo, Merlot, Garnacha, Graciano, and Mencia wines. The most abundant compounds in Cabernet Sauvignon were Mv-3-glu, Mv-3-ace, Mv-3-cum, Dp-3-glu, Pt-3-glu, and Pn-3-glu. Interestingly, in Tempranillo samples, the content of 3-O-glucosides (except Mv-3-glu) was twice or higher than in Cabernet Sauvignon. Mv-3-glu $>$ Mv-3-ace $>$ Pn-3-glu $>$ Mv-3-cum $>$ Dp-3-glu $>$ Pt-3-glu were the most abundant anthocyanins in Merlot. Merlot had the highest concentrations of Cy-3-glu (> Pn-3-glu > Pn-3-cum) of all studied wines. Instead, Garnacha showed the highest concentration of Mv-3-glu and low concentrations of other anthocyanins. Finally, in Garciano wines the distribution of anthocyanins was: Mv-3-glu $>$ Pn-3-ace $>$ Mv-3-cum $>$ Pt-3-glu $>$ Dp-3-glu. Gonzalez-Neves et al. [50] were mainly focused on the content of 3-O-glucosides in Merlot, Syrah, and Tannat wines. Merlot samples showed the same anthocyanins pattern as previously discussed. It also had the highest content of total acetyl glucosides among all wines. Both in Syrah and Tannat, the most abundant compound was Mv-3-glu and the least abundant was Cy-3-glu. The pattern of 3-O-glucosides in Syrah were Pn-3-glu > Pt-3-glu $>$ Dp-3-glu and in Tannat Pt-3-glu > Dp-3-glu > Pn-3-glu. Garcia-Beneytez et al. [51] have shown that Dp-3-ace, Cy-3-ace, and Pt-3-ace were present only in Cabernet Sauvignon, Merlot, and Monastrell wines and were not found in Alicante Bouschet, Bobal, Garnacha, and Tempranillo. Teinturier varieties (including Alicante Bouschet, Morrastel Bouschet and Petit Bouschet) contain peonidin 3-O-glucoside (as the major anthocyan) not only in skin cells but also in the flesh according to the same study.

Muccillo et al. [52] analyzed the trend of the malvidin-3-O-glucoside (Mv-3-glu), 3-acetylglucoside (Mv-3-ace), and 3-p-coumarylglucoside (Mv-3-cum) in studied red wines. In Piedirosso, Cabernet Sauvignon, Merlot, and Lingua di Femmina wines, the distributions according to the concentrations were $\mathrm{Mv}$-3-glu $>\mathrm{Mv}$-3-ace $>\mathrm{Mv}$-3-cum and differed from Aglianico wines, where anthocyanins were distributed as Mv-3-glu $>$ Mv-3-cum $>$ Mv-3-ace. This observation came with an agreement of previous studies related with the anthocyanin content of these wines. Kumšta et al. [53] have found that a combination of delphinidin-3-O-glucoside, malvidin-3-O-glucoside, peonidin-3-O-coumarylglucoside, and delphinidin-3-O-acetylglucoside can cluster wines made from Blaufränkisch, Blauer Portugieser, and Saint Laurent grapes without influence of the geographical origin. Furthermore, the highest concentrations of malvidin derivatives were found in Blauer 
Portugieser, while Blaufränkisch was noted to the markedly lower content of acylated anthocyanins than glycosidic ones.

Moreover, some important studies on the anthocyanin profiles of grape skins concerning Nebbiolo and Cabernet Sauvignon varieties were done [54,55]. Both investigations agreed that the most abundant anthocyanin in Nebbiolo grape skins was peonidin-3-O-glucoside. The percentage between disubstituted anthocyanins and Pn-3-glu was affected by growing location [55]. The loss of disubstituted anthocyanins during the skin maceration was observed in both Nebbiolo and Cabernet Sauvignon (lesser than in Nebbiolo) grapes, as well as the increment of trisubstituted anthocyanins [55]. During the vinification, Pn-3-glu tends to oxidize, and its concentration decreases. However, Pn-3-glu is the second most present anthocyanin in Nebbiolo wine after Mv-3-glu [56].

Pisano et al. [14] have noticed that malvidin-3-O-glucoside, malvidin-3-(6-O-acetylglucoside), and malvidin-3-O-glucoside-4-vinylguaiacol (or malvidin-3-(6-O-p-coumaroylglucoside) are good discriminants between Aspiran, Bonarda, Cabernet Sauvignon, Malbec, Merlot, Sangiovese, Syrah, and Tempranillo wines from Argentina. A major study of 11 single-cultivar Italian red wines was performed [57] and possible chemical markers for seven varieties were found: hydroxycinnamates for Cannonau wines; anthocyanins-Teroldego; flavan-3-ols-Aglianico, Nerello, Nebbiolo; flavan-3-ols and flavonols-Sangiovese; amino acids and metabolites that contain nitrogen-Primitivo. In addition, ratios between specific anthocyanins $[58,59]$ or their combination with other secondary metabolites [60] were applied. Using a set of anthocyanins, their proportions and phenolic and organic acids abundances were used to build a principal component analysis (PCA) model, in which the studied grape varieties (Cabernet Sauvignon, Feteasca Neagra, Mamaia, and Merlot) were $100 \%$ classified correctly, except for Pinot Noir, which was classified only in $87.50 \%$ of cases, since the phenolic profiles of Pinot Noir and Feteasca Neagra were quite similar [58]. The study [59] emphasized that both Corvina Veronese and Negro Amaro wines have high levels of disubstituted compounds and low levels of acyl derivatives. These wines can be chemically distinguished from each other with laricitrin, syringetin (that are low in Corvina Veronese), quercetin, and kaempferol (that are high in Negro Amaro). The anthocyanic profile of Primitivo showed high contents of $\left(3^{\prime}, 4^{\prime}, 5^{\prime}\right)$ trisubstituted flavonoids, such as laricitrin, myricetin, and syringetin, as well as a high content of trisubstituted anthocyanins, such as petunidin and malvidin derivatives and a lower content of isorhamnetin and kaempferol derivatives. De Rosso et al. [60] presented the application of indexes of laricitrin, delphinidin, and petunidin for the detection of Primitivo and Negro Amaro in the wine blends.

In addition, other researchers suggested combining the profile of anthocyanins with the content of phenolic acids [61], flavonols [18], phenolic acids, and flavan-3-ols [62]. Based on the statistical evaluation, flavan-3-ols alone [63] or combined with phenolic acids [64] and condensed proanthocyanidins $[65,66]$ were found to distinguish well Graciano, Tempranillo, Cabernet Sauvignon, Cabernet Franc, Carménère, Merlot Pinotage, Syrah, and Sangiovese grape varieties. Interestingly, a set of specific markers was observed for Carménère and Merlot wines produced in Chile: a ratio of total quercetin and total myricetin combined with the concentration of myricetin itself [29].

The fingerprint composed by phenolic acids, flavonoids, tannins, and stilbenes can be used for the classification on the basis of mainly local grape variety in terms of producing country, as it was recently shown for Croatian wines [19]. In this study, taxifolin and peonidin acetylglucoside were differentiating red wines (Cabernet Sauvignon, Merlot, Plavac mali, Teran), whereas cis-piceid was efficient in differentiating white monovarietal wines (Chardonnay, Graševina, Malvazija Istarska, Maraština, Muscat Blanc, Pošip). Martelo-Vidal et al. [38] applied chemometric methods to Rías Baixas and Ribeira Sacra wines that determined significantly high concentrations of malvin in Rías Baixas and trans-resveratrol in Ribeira Sacra. These phenolics, together with syringic acid, oenin, (+)-catechin, (-)-epicatechin, and quercetin, proved to be a good set for the sample discrimination. Another study was mainly focused on the local grape varieties Vranac, Kratosija, and wines from Cabernet Sauvignon grapes [67]. It showed that Vranac distinguished in high contents of anthocyanins, Kratošija-hydroxycinnamic acids and Cabernet Sauvignon-flavan-3-ols. Ragusa et al. [39] reported 
that Negroamaro and Primitivo can be differentiated by syringic acid, hydroxytyrosol (that are high in Primitivo), and trans-resveratrol (high in Negroamaro). Finally, Salvatore et al. [68] described phenolic profiles of Lambrusco di Sorbara, Lambrusco Salamino di Santa Croce, and Lambrusco Grasparossa di Castelvetro. Lambrusco di Sorbara had significant concentrations of $p$-coumaric and caffeic acids, Lambrusco Salamino di Santa Croce—gallic acid, Lambrusco Grasparossa di Castelvetro-myricetin and quercitin.

Even though some studies $[1,9,32,69,70]$ did not identify specific phenolic markers and used spectral areas in their quality control assessment, the spectroscopic analyses showed good discrimination among wines made with both white and red grape varieties with a high sample variability. However, Magdas et al. [71] have reported a strong overlap between Chardonnay and Sauvignon samples. The final separation using a linear discriminant analysis among all four cultivars (Riesling and Pinot Gris as well) was only $84 \%$.

A new class of macrocyclic proanthocyanidins was recently discovered in grapes and wine. The congeners of cyclic (crown) oligomeric proanthocyanidins (procyanidins and prodelphinidins) are currently under study to evaluate their suitability as potential chemical markers for white and red grape varieties [72-74]. The ratios of cyclic compounds versus the sum of cyclic and non-cyclic congeners per number of monomer units showed an interesting relationship with the grape variety (red or white).

In addition, new acylated flavonols were recently identified [75] in Tannat, Marselan, Syrah grapes, and wines produced in the Southern Uruguay. They are acetylated derivatives of the flavonol glucosides containing methoxylated aglycones, such as isorhamnetin, laricitrin, and syringetin, and they might be used for the quality and authenticity assessment. 
Table 1. Chemical markers proposed to differentiate wines produced with different grape varieties.

\begin{tabular}{|c|c|c|c|c|c|c|}
\hline Grape Variety & Type of Wine & Chemical Markers & Role of Chemical Markers & Analytical Method & Statistical Method & References \\
\hline $\begin{array}{l}\text { Aurelius, Chardonnay, Müller } \\
\text { Thurgau, Moravian Muscat, } \\
\text { Hibernal, Malverina, Merzling }\end{array}$ & W & $\begin{array}{l}p \text {-coutaric acid, caftaric } \\
\text { acid, protocatechuic and } \\
\text { syringic acid; } \\
\text { (+)-catechin, } \\
\text { (-)-epicatechin }\end{array}$ & $\begin{array}{l}\text { Different set of specific } \\
\text { phenolic acids (absolute } \\
\text { concentrations) }\end{array}$ & HPLC-DAD & CVA & [42] \\
\hline $\begin{array}{l}\text { Chardonnay, Riesling, } \\
\text { Sauvignon Blanc }\end{array}$ & W & $\begin{array}{c}\text { Shikimic acid, ferulic acid, } \\
\text { trans-caffeic acid, } \\
\text { epicatechin }\end{array}$ & $\begin{array}{l}\text { The most significant loadings } \\
\text { in the NMR spectrum }\end{array}$ & NMR & LDA & [43] \\
\hline $\begin{array}{c}\text { Chardonnay, Muscat, Cabernet } \\
\text { Sauvignon, Cienna, Dolcetto, } \\
\text { Durif, Merlot, Petit Verdot, } \\
\text { Pinot Noir, Primitivo, Syrah, } \\
\text { Zinfandel }\end{array}$ & $\mathrm{W}, \mathrm{R}$ & $\begin{array}{l}\text { Caffeic acid, galic acid, } \\
\text { rutin, trans-resveratrol }\end{array}$ & $\begin{array}{c}\text { Different content of caffeic acid, } \\
\text { gallic acid and rutin in wine } \\
\text { samples; lack of } \\
\text { trans-resveratrol in } \\
\text { Chardonnay and Muscat }\end{array}$ & HPTLC & $\begin{array}{l}\text { HCA, SVD, } \\
\text { PCA, ANN }\end{array}$ & [10] \\
\hline Müller-Thurgau, Riesling & W & $\begin{array}{l}\text { Amino and organic acids, } \\
\text { phenolic acids, flavonoids } \\
\text { and stilbenes }\end{array}$ & $\begin{array}{l}\text { High concentration of } \\
\text { quercetin, kaempferol, } \\
\text { resveratrol in Müller-Thurgau; } \\
\text { high (+)-catechin, } \\
\text { (-)-epicatechin, caftarate and } \\
\text { coutarate in Riesling }\end{array}$ & NMR & PLS & [44] \\
\hline $\begin{array}{l}\text { Lemberger, Pinot Blanc, Pinot } \\
\text { Gris, Müller-Thurgau, Riesling, } \\
\text { Gewürztraminer, Pinot Noir }\end{array}$ & $\mathrm{W}, \mathrm{R}$ & $\begin{array}{l}\text { Phenolic compounds } \\
\text { (quercetin, catechin, } \\
\text { resveratrol, gallate) }\end{array}$ & $\begin{array}{l}\text { Specific fingerprints due to } \\
\text { variety, origin, vintage, } \\
\text { physiological state, and } \\
\text { technological treatment }\end{array}$ & NMR & $\begin{array}{l}\text { CV, LDA, } \\
\text { MANOVA, MC, } \\
\text { NCM, PCA, }\end{array}$ & [45] \\
\hline Passerina, Verdicchio & W & $\begin{array}{l}\text { Various phenolic } \\
\text { compounds }\end{array}$ & $\begin{array}{l}\text { High concentration of tyrosol, } \\
\text { quercetin and glucuronide in } \\
\text { Passerina; and hydroxytyrosol, } \\
\text { caffeic, caftaric, coumaric and } \\
\text { 2-S-glutathionylcaftaric acids } \\
\text { (including their esters) in } \\
\text { Verdicchio }\end{array}$ & HPLC-DAD & PCA & [46] \\
\hline $\begin{array}{c}\text { Bianco d'Alessano, } \\
\text { Chardonnay, Falanghina, } \\
\text { Fiano, Malvasia, Moscato, } \\
\text { Negroamaro, Verdecca, } \\
\text { Malvasia Nera, Primitivo, } \\
\text { Susumaniello }\end{array}$ & W, Rs, R & $\begin{array}{l}\text { Gallic acid, syringic acid, } \\
\text { luteolin, quercitin, } \\
\text { hydroxytyrosol, } \\
\text { trans-resveratrol }\end{array}$ & $\begin{array}{l}\text { Specific set of absolute } \\
\text { concentrations of phenolics }\end{array}$ & HPLC-DAD & GDA, PCA & [23] \\
\hline
\end{tabular}


Table 1. Cont.

\begin{tabular}{|c|c|c|c|c|c|c|}
\hline Grape Variety & Type of Wine & Chemical Markers & Role of Chemical Markers & Analytical Method & Statistical Method & References \\
\hline $\begin{array}{l}\text { Blaufränkisch, Blauer } \\
\text { Portugieser, Pinot Noir, Sankt } \\
\text { Laurent, Zweigeltrebe }\end{array}$ & Rs & $\begin{array}{l}\text { Caftaric acid, coumaric } \\
\text { acid, ferrulic acid, } \\
\text { catechin, } \\
\text { malvidin-3-O-glucoside, } \\
\text { epicatechin, cis- and } \\
\text { trans-resveratrol }\end{array}$ & $\begin{array}{l}\text { Specific set of absolute } \\
\text { concentrations of phenolics }\end{array}$ & HPLC-DAD & $\begin{array}{c}\text { Box and } \\
\text { Whisker Plot }\end{array}$ & [37] \\
\hline $\begin{array}{c}\text { Cabernet Sauvignon, Feteasca } \\
\text { Neagra, Mamaia, Merlot, } \\
\text { Pinot Noir }\end{array}$ & $\mathrm{R}$ & $\begin{array}{l}\text { Anthocyanins, } \\
\text { anthocyanins ratios, } \\
\text { phenolic and }\end{array}$ & $\begin{array}{l}\text { Different contents (expressed in } \\
\text { malvidin-3-O-glucoside) of } \\
\text { acylated malvidin and acylated } \\
\text { malvidin/malvidin in } \\
\text { combination with acids in } \\
\text { different grape varieties }\end{array}$ & $\begin{array}{l}\text { HPLC-PDA, } \\
\text { NMR }\end{array}$ & LDA, PCA & [58] \\
\hline $\begin{array}{l}\text { Aspiran, Bonarda, Cabernet } \\
\text { Sauvignon, Malbec, Merlot, } \\
\text { Sangiovese, Syrah, Tempranillo }\end{array}$ & $\mathrm{R}$ & $\begin{array}{c}\text { Malvidin-3-O-glucoside, } \\
\text { malvidin-3-(6-O- } \\
\text { acetylglucoside), } \\
\text { malvidin-3-O- } \\
\text { glucoside-4-vinylguaiacol }\end{array}$ & $\begin{array}{l}\text { Absolute concentration (not } \\
\text { specified according grape } \\
\text { variety) }\end{array}$ & HPLC-MS & MCR-ALS, D-UPLS & [14] \\
\hline $\begin{array}{c}\text { Aglianico (Ag), Cannonau (Ca), } \\
\text { Corvina, Montepulciano, } \\
\text { Nebbiolo (Nb), Nerello (Nr), } \\
\text { Primitivo (Pr), Raboso, } \\
\text { Sagrantino, Sangiovese (Sa), } \\
\text { Teroldego (Te) }\end{array}$ & $\mathrm{R}$ & $\begin{array}{c}\text { Hydroxycinnamates }(\mathrm{Ca}), \\
\text { Anthocyanins }(\mathrm{Te}), \\
\text { flavan-3-ols (Ag, } \mathrm{Sa}, \mathrm{Nb}, \\
\mathrm{Nr} \text { ), flavonols (Sa), amino } \\
\text { acids (Pr) }\end{array}$ & $\begin{array}{l}\text { Specific classes of markers } \\
\text { through grape varieties }\end{array}$ & UHPLC-QTOF MS & ANOVA, PCA & [56] \\
\hline $\begin{array}{c}\text { Corvina Veronese, } \\
\text { Negroamaro, Primitivo, } \\
\text { Raboso Piave }\end{array}$ & $\mathrm{R}$ & $\begin{array}{l}\text { Ratios of anthocyanins, } \\
\text { various phenolic } \\
\text { compounds, volatile } \\
\text { profile }\end{array}$ & $\begin{array}{l}\text { High concentration of } \\
\text { disubstituted compounds and } \\
\text { lower acyl derivatives in } \\
\text { Corvina and Negroamaro; high } \\
\text { content of trisubstituted } \\
\text { flavonoids in Primitivo; and } \\
\text { anthocyanic and } \\
\text { non-anthocyanic acyl } \\
\text { derivatives in Raboso }\end{array}$ & UHPLC-Q/TOF & $\begin{array}{l}\text { HCA, PCA, } \\
\text { Tukey test }\end{array}$ & [59] \\
\hline Amarone, Recioto, Primitivo & $\mathrm{R}$ & $\begin{array}{l}\text { Ratios laricitrin, } \\
\text { delphinidin, and } \\
\text { petunidin }\end{array}$ & $\begin{array}{l}\text { Ratios have identified the use } \\
\text { of } 10 \% \text { Primitivo in wine } \\
\text { blends }\end{array}$ & UHPLC-Q/TOF & $\begin{array}{l}\text { HCA, Heat maps, } \\
\text { Tukey test }\end{array}$ & [60] \\
\hline
\end{tabular}


Table 1. Cont.

\begin{tabular}{|c|c|c|c|c|c|c|}
\hline Grape Variety & Type of Wine & Chemical Markers & Role of Chemical Markers & Analytical Method & Statistical Method & References \\
\hline $\begin{array}{c}\text { Aspiran, Bonarda, Cabernet } \\
\text { Sauvignon, Malbec, Merlot, } \\
\text { Sangiovese, Syrah, Tempranillo }\end{array}$ & $\mathrm{R}$ & $\begin{array}{l}\text { Phenolic acids and } \\
\text { anthocyanins }\end{array}$ & $\begin{array}{l}\text { Differentiation of Malbec; less } \\
\text { effective for geographical } \\
\text { origin }\end{array}$ & HPLC-DAD & MCR-ALS & [61] \\
\hline $\begin{array}{l}\text { Baboso, Castellana, Listán } \\
\text { Negro, Listán Prieto, Merlot, } \\
\text { Negramoll, Ruby Cabernet, } \\
\text { Syrah, Tintilla, Vijariego. }\end{array}$ & $\mathrm{R}$ & $\begin{array}{c}\text { Flavonols and } \\
\text { anthocyanins }\end{array}$ & $\begin{array}{l}\text { Lower or higher absolute } \\
\text { concentrations }\end{array}$ & HPLC-DAD & $\begin{array}{l}\text { ANOVA, PCA, } \\
\text { Pearson coefficient }\end{array}$ & [18] \\
\hline $\begin{array}{c}\text { Bonarda, Cabernet Sauvignon, } \\
\text { Malbec, Merlot, Syrah, } \\
\text { Tempranillo }\end{array}$ & $\mathrm{R}$ & $\begin{array}{l}\text { Phenolic acids, } \\
\text { flavan-3-ols, } \\
\text { anthocyanins }\end{array}$ & $\begin{array}{l}\text { Specific set of the absolute } \\
\text { concentrations of phenolics }\end{array}$ & HPLC-MS & ANOVA, HSD & [62] \\
\hline $\begin{array}{c}\text { Cabernet Sauvignon, Graciano, } \\
\text { Tempranillo }\end{array}$ & $\mathrm{R}$ & Flavan-3-ols & $\begin{array}{l}\text { Absolute concentrations of } \\
\text { phenolics }\end{array}$ & HPLC-DAD/ESI-MS & - & [63] \\
\hline $\begin{array}{l}\text { Cabernet Sauvignon, } \\
\text { Pinotage, Syrah }\end{array}$ & $\mathrm{R}$ & $\begin{array}{l}\text { Flavan-3-ols and } \\
\text { phenolic acids }\end{array}$ & $\begin{array}{l}\text { Specific set of the absolute } \\
\text { concentrations of phenolics }\end{array}$ & HPLC-DAD & $\begin{array}{l}\text { Multiple linear } \\
\text { regression analysis }\end{array}$ & [64] \\
\hline $\begin{array}{c}\text { Cabernet Sauvignon, Cabernet } \\
\text { Franc, Carménère, } \\
\text { Merlot, Syrah }\end{array}$ & $\mathrm{R}$ & $\begin{array}{l}\text { Flavan-3-ols and } \\
\text { phenolic acids }\end{array}$ & $\begin{array}{l}\text { Absolute concentrations of } \\
\text { phenolics }\end{array}$ & HPLC-DAD/PDA & PCA & [65] \\
\hline $\begin{array}{l}\text { Cabernet Franc, Merlot, } \\
\text { Sangiovese, Syrah }\end{array}$ & $\mathrm{R}$ & Flavan-3-ols, tannins & $\begin{array}{l}\text { Lower or higher absolute } \\
\text { concentrations }\end{array}$ & HPLC-DAD-MS & $\begin{array}{l}\text { ANOVA, PCA, } \\
\text { Tukey test }\end{array}$ & [64] \\
\hline Carménère, Merlot & $\mathrm{R}$ & $\begin{array}{l}\text { Ratio of total } \\
\text { quercetin/total myricetin } \\
\text { and concentration of } \\
\text { myricetin }\end{array}$ & Different ratio contents & HPLC-DAD-ESI-MS ${ }^{n}$ & DA, PCA & [29] \\
\hline $\begin{array}{c}\text { Chardonnay, Graševina, } \\
\text { Malvazija Istarska, Maraština } \\
\text { Muscat Blanc; Pošip, Cabernet } \\
\text { Sauvignon, Merlot, Plavac } \\
\text { mali, Teran }\end{array}$ & $\mathrm{W}, \mathrm{R}$ & $\begin{array}{c}\text { Phenolic acids, } \\
\text { flavonoids, tannins and } \\
\text { stilbenes }\end{array}$ & $\begin{array}{l}\text { Specific concentrations for each } \\
\text { wine; the content of } \\
\text { cis-piceid-discriminant for } \\
\text { white wines, peonidin } \\
\text { 3-(6"-acetyl)-glucoside and } \\
\text { taxifolin-for red wines }\end{array}$ & $\begin{array}{l}\text { UHPLC-QqQ- } \\
\text { MS/MS }\end{array}$ & $\begin{array}{l}\text { ANOVA, LSA, } \\
\text { SLDA }\end{array}$ & [19] \\
\hline
\end{tabular}


Table 1. Cont.

\begin{tabular}{|c|c|c|c|c|c|c|}
\hline Grape Variety & Type of Wine & Chemical Markers & Role of Chemical Markers & Analytical Method & Statistical Method & References \\
\hline Rías Baixas, Ribeira Sacra & $\mathrm{R}$ & $\begin{array}{l}\text { Syringic acid, malvin, } \\
\text { oenin, (+)-catechin, } \\
\text { (-)-epicatechin, quercetin, } \\
\text { trans-resveratrol }\end{array}$ & $\begin{array}{c}\text { Specific absolute } \\
\text { concentrations; high amounts } \\
\text { of trans-resveratrol in Ribeira } \\
\text { Sacra and malvin in Rías Baixas }\end{array}$ & HPLC-DAD & $\begin{array}{l}\text { LDA, PCA, } \\
\text { SIMCA, SVM }\end{array}$ & [38] \\
\hline $\begin{array}{l}\text { Vranac, Kratošija, } \\
\text { Cabernet Sauvignon }\end{array}$ & $\mathrm{R}$ & $\begin{array}{c}\text { Phenolic acids, } \\
\text { flavonoids, tannins and } \\
\text { stilbenes }\end{array}$ & $\begin{array}{c}\text { Vranac-high content of } \\
\text { anthocyanins; Kratošija-high } \\
\text { content of hydroxycinnamic } \\
\text { acids; Cabernet } \\
\text { Sauvignon-high content of } \\
\text { flavan-3-ols and low content of } \\
\text { stilbenes }\end{array}$ & HPLC-DAD & ANOVA, LSD & [67] \\
\hline Negroamaro, Primitivo & $\mathrm{R}$ & $\begin{array}{l}\text { Gallic acid, syringic acid, } \\
\text { catechin, quercetin, } \\
\text { hydroxytyrosol, } \\
\text { trans-resveratrol }\end{array}$ & $\begin{array}{l}\text { Significant differences of } \\
\text { amounts of syringic acid and } \\
\text { hydroxytyrosol in Primitivo, } \\
\text { trans-resveratrol in } \\
\text { Negroamaro }\end{array}$ & HPLC-DAD & $\begin{array}{l}\text { MVA, PCA, } \\
\text { OPLS-DA, SIMCA }\end{array}$ & [39] \\
\hline $\begin{array}{c}\text { Lambrusco Sorbara, } \\
\text { Lambrusco Salamino di Santa } \\
\text { Croce, Lambrusco Grasparossa } \\
\text { di Castelvetro }\end{array}$ & $\mathrm{R}$ & $\begin{array}{c}\text { Caffeic acid, galic acid, } \\
\text { p-coumaric acid, syringic } \\
\text { acid, catechin, miricetin, } \\
\text { quercitin }\end{array}$ & $\begin{array}{l}p \text {-coumaric and caffeic acids } \\
\text { describes Sorbara; gallic } \\
\text { acid-Salamino; myricetin and } \\
\text { quercitin—Grasparossa }\end{array}$ & HPLC-DAD & PCA, PLS & [68] \\
\hline $\begin{array}{l}\text { Vilana, Dafni, } \\
\text { Kotsifali, Mandilari }\end{array}$ & $\mathrm{W}, \mathrm{R}$ & $\begin{array}{l}\text { Spectral regions from } \\
1800 \text { to } 1500 \mathrm{~cm}^{-1} \text { and } \\
\text { from } 1300 \text { to } 900 \mathrm{~cm}^{-1}\end{array}$ & $\begin{array}{l}\text { Different fingerprints } \\
\text { (band intensity) }\end{array}$ & FT-IR & LDA & [32] \\
\hline $\begin{array}{c}\text { Cabernet Sauvignon, Feteasca } \\
\text { Neagra, Mamaia, Merlot, } \\
\text { Pinot Noir }\end{array}$ & $\mathrm{R}$ & $\begin{array}{l}\text { Phenolics compounds in } \\
\text { the } 250-600 \mathrm{~nm} \text { region }\end{array}$ & $\begin{array}{l}\text { Different fingerprints } \\
\text { (band intensity) }\end{array}$ & UV-Vis & LDA, PCA, PLS-DA & [1] \\
\hline $\begin{array}{c}\text { Chardonnay, Feteasca Regala, } \\
\text { Sauvignon Blanc }\end{array}$ & W & $\begin{array}{l}\text { Signals at } 1245,1575 \text { and } \\
\qquad 1581 \mathrm{~cm}^{-1}\end{array}$ & $\begin{array}{l}\text { Different fingerprints } \\
\text { (band intensity) }\end{array}$ & SERS & LDA & [69] \\
\hline $\begin{array}{l}\text { Feteasca Regala, } \\
\text { Sauvignon Blanc }\end{array}$ & W & $\begin{array}{l}\text { Mainly phenolic acids at } \\
-767,-543,-530,-653 \\
1608 \text { and }-881 \mathrm{~cm}^{-1}\end{array}$ & $\begin{array}{l}\text { Different fingerprints } \\
\quad \text { (band intensity) }\end{array}$ & FT-Raman & SLDA & [70] \\
\hline
\end{tabular}


Table 1. Cont.

\begin{tabular}{|c|c|c|c|c|c|c|}
\hline Grape Variety & Type of Wine & Chemical Markers & Role of Chemical Markers & Analytical Method & Statistical Method & References \\
\hline $\begin{array}{c}\text { Sangiovese, Nebbiolo, } \\
\text { Aglianico, Nerello Mascalese, } \\
\text { Primitivo, Raboso, Cannonau, } \\
\text { Teroldego, Sagrantino, } \\
\text { Montepulciano, Corvina }\end{array}$ & $\mathrm{R}$ & Tannins & $\begin{array}{l}\text { Different fingerprints } \\
\text { (band intensity) }\end{array}$ & MIR & $\begin{array}{l}\text { LDA, PCA, } \\
\text { SIMCA, SVM }\end{array}$ & [9] \\
\hline $\begin{array}{l}\text { Chardonnay, Pinot Gris, } \\
\text { Riesling, Sauvignon }\end{array}$ & W & $\begin{array}{l}\text { Flavonoids, tannins, } \\
\text { stilbenes }\end{array}$ & $\begin{array}{l}\text { Different fingerprints } \\
\text { (band intensity) }\end{array}$ & FT-Raman & LDA & [71] \\
\hline $\begin{array}{l}\text { Chardonnay, Gewürztraminer, } \\
\text { Sauvignon Blanc, Lagrein, } \\
\text { Cabernet Franc, Cabernet } \\
\text { Sauvignon, Merlot, Pinot Nero }\end{array}$ & W, R & $\begin{array}{l}\text { Ratios of cyclic } \\
\text { prodelphinidins and the } \\
\text { sum of cyclic and } \\
\text { non-cyclic } \\
\text { prodelphinidins }\end{array}$ & $\begin{array}{c}\text { High or low ratios according } \\
\text { to variety }\end{array}$ & HPLC-DAD-HRMS/MS & PCA & [72] \\
\hline $\begin{array}{l}\text { Chardonnay, Gewürztraminer, } \\
\text { Sauvignon Blanc, Lagrein, } \\
\text { Cabernet Franc, Cabernet } \\
\text { Sauvignon, Merlot, Pinot Nero }\end{array}$ & $\mathrm{W}, \mathrm{R}$ & $\begin{array}{c}\text { Ratios of cyclic } \\
\text { procyanidins and the } \\
\text { sum of cyclic and } \\
\text { non-cyclic procyanidins }\end{array}$ & $\begin{array}{c}\text { High or low ratios according } \\
\text { to variety }\end{array}$ & HPLC-DAD-HRMS/MS & PCA & [73] \\
\hline \multicolumn{7}{|c|}{ 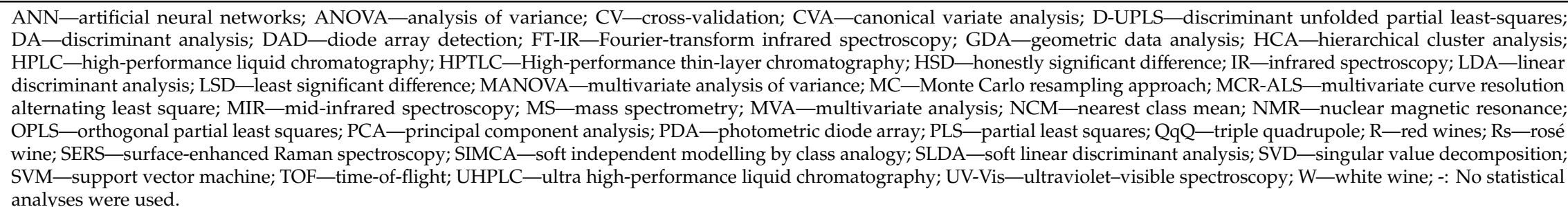 } \\
\hline
\end{tabular}


Table 2. Anthocyanins proposed to differentiate wines produced with different grape varieties of Vitis vinifera, measured by LC.

\begin{tabular}{|c|c|c|}
\hline Grape Variety & Chemical Markers and Their Role & References \\
\hline Tempranillo & $\begin{array}{l}\text { Mv-3-glu }>\text { Pt-3-glu }>\text { Dp-3-glu }>\text { Pn-3-glu }>\text { Cy-3-glu, Mv-3-ace }>\text { Pt-3-ace }>\text { Pn-3-ace }>\text { Dp-3-ace } \\
>\text { Cy-3-ace, trans-Mv-3-cum }>\text { Dp-3-cum }>\text { Pn-3-cum }>\text { cis-Mv-3-cum }>\text { Cy-3-cum, Mv-caf }>\text { Pn-caf }\end{array}$ & \multirow{6}{*}{ [22] } \\
\hline Tortosi & All present, except: Cy-3-glu, Pt-3-ace, Pn-3-ace, Dp-3-cum, Pn-caf & \\
\hline Bobal & Low concentrations of anthocyanins, Pt-3-ace, Pn-3-ace and Pn-caf were not present & \\
\hline Moravia Agria & Cy-3-ace, Mv-3-ace, cis-Mv-3-cum, Pn-3-cum, trans-Mv-3-cum and Mv-caf only & \\
\hline Moravia Dulce & $\begin{array}{l}\text { Dp-3-glu, Pt-3-glu, Pn-3-glu, Mv-3-glu, Cy-3-ace, Mv-3-ace, cis-Mv-3-cum, Pn-3-cum, } \\
\text { trans-Mv-3-cum only }\end{array}$ & \\
\hline Rojal & Pn-3-glu, Mv-3-glu, Cy-3-ace only & \\
\hline Cabernet Sauvignon & Mv-3-glu, Mv-3-ace, Mv-3-cum, Dp-3-glu, Pt-3-glu and Pn-3-glu & \multirow{5}{*}{ [27] } \\
\hline Garnacha & Highest concentration of Mv-3-glu and low concentrations of other anthocyanins & \\
\hline Graciano & Mv-3-glu $>$ Pn-3-ace $>$ Mv-3-cum $>$ Pt-3-glu $>$ Dp-3-glu & \\
\hline Merlot & Mv-3-glu $>$ Mv-3-ace $>$ Pn-3-glu $>$ Mv-3-cum $>$ Dp-3-glu $>$ Pt-3-glu & \\
\hline Tempranillo & Higher content of 3-O-glucosides (except Mv-3-glu) than Cabernet Sauvignon & \\
\hline Syrah & Pn-3-glu $>$ Pt-3-glu $>$ Dp-3-glu & \multirow{2}{*}{ [50] } \\
\hline Tannat & Pt-3-glu > Dp-3-glu > Pn-3-glu & \\
\hline Alicante Bouschet, Cabernet Sauvignon, Merlot, Monastrel Bouschet & $\begin{array}{l}\text { Various concentrations of Dp-3-ace, Cy-3-ace and Pt-3-ace; Pn-3-glu is a major anthocyanin only } \\
\text { for Bouschet varieties }\end{array}$ & \multirow{2}{*}{ [51] } \\
\hline Bobal, Garnacha, Petit Bouschet, Tempranillo & Not present: Dp-3-ace, Cy-3-ace and Pt-3-ace; Pn-3-glu is a major anthocyanin & \\
\hline Cabernet Sauvignon, Lingua di Femmina, Merlot, Piedirosso & Specific absolute concentrations distribution: Mv-3-glu $>$ Mv-3-ace $>$ Mv-3-cum & \multirow{2}{*}{ [52] } \\
\hline Aglianico del Taburno, Aglianico del Vulture, Aglianico di Taurasi & Specific absolute concentrations distribution: Mv-3-glu $>$ Mv-3-cum $>$ Mv-3-ace & \\
\hline Blaufränkisch, Blauer Portugieser, Saint Laurent & Specific set of absolute concentrations: Dp-3-glu, Mv-3-glu, Dp-3-ace for each variety & [53] \\
\hline Nebbiolo & Mv-3-glu > Pn-3-glu (Pn-3-glu is half of Mv-3-glu concentration) & [56] \\
\hline
\end{tabular}




\subsubsection{Non Vitis Vinifera Grape Species}

The most cultivated grapes used to produce wines belong to the Vitis vinifera grape species, since the regulations of the European Union allow only this species to be used for a commercial purpose [38]. Currently, there are only a few studies dealing with the evaluation of phenolic profile of hybrids and other species than Vitis vinifera (Table 3). It has been noticed that the anthocyanin profile of non Vitis vinifera grapes consist of 3,5-diglucosidic forms [36]. Small concentrations of these compounds can be also found in some Vitis vinifera grapes and wines [76-79]. In 2012, the International Organization of Vine and Wine (OIV) published a list of maximum acceptable limits of various chemical compounds, including the amount of malvidin-3,5-diglucoside (max. $15 \mathrm{mg} / \mathrm{L}$ ) that can be used as a chemical marker of non Vitis vinifera grape species [80].

The content of the anthocyanin 3,5-diglucosides proved to be a good chemical marker for the differentiation of Vitis vinifera red wines and Isabel (Vitis vinifera $\times$ Vitis labrusca hybrid) wines by Nixdorf et al. [81]. Isabel is a hybrid grape cultivar that covers half of Brazilian grape production, and it is used to produce table wine, grape juice, and other drinks and food products.

Common wine adulteration cases in Poland are based on labeling wines made from hybrid Rondo (Vitis amurensis $\times$ Vitis vinifera) grapes as made with the Zweigelt (Vitis vinifera) grapes. To provide tools to discover these frauds, Stój et al. [5] analyzed the content of phenolic acids, flavonoids, tannins, and stilbenes of wines from both grape varieties. The 3,5-diglucosidic anthocyanins, Rondo wines had higher amounts of gallic acid and stilbenes (trans-piceid and cis-piceid). Spine grape (Vitis davidii Foex) is one of the main wild grape species growing in East Asia, and it is used mainly for red and white wine production [82]. The wine made from spine grapes contains malvidin-3,5-diglucoside, syringetin-3-O-glucoside, dihydroquercetin-3-hexoside, and coutaric acid [82]. Native Chinese species, such as Vitis amurensis and Vitis davidii were studied by Li et al. [83]. Wines made from Vitis davidii grapes were also characterized by higher concentrations of hydroxycinnamic acids than hydroxybenzoic acids and distinguished by the high contents of kaempferol-3-O-glucoside and quercetin-3-O-rhamnoside, in comparison with other grape species. Burns et al. [84] used the proportion of mono-glucoside and acetylated anthocyanins to distinguish Cabernet Sauvignon wines from the hybrid grape varieties from the North America: Baco, Seybel, Clinton, Jacquez, and Othello. Gougeon et al. [85] investigated the authenticity (origin and vintage) of Cabernet Sauvignon (Vitis vinifera) and Beihong (Vitis vinifera and Vitis amurensis). Using the content of various phenolic compounds (mainly phenolic acids), good separations between samples were observed in terms of grape variety and terroir. Unfortunately, wines were not clustered according to the vintage. 
Table 3. Chemical markers for the wine authenticity and quality control in terms of non-Vitis vinifera grape species or hybrid species.

\begin{tabular}{|c|c|c|c|c|c|c|}
\hline Grape Species & Type of Wine & Chemical Markers & $\begin{array}{c}\text { Role of Chemical } \\
\text { Marker }\end{array}$ & Analytical Method & Statistical Method & References \\
\hline $\begin{array}{l}\text { Hybrid grape Isabel (Vitis vinifera } \\
\times \text { Vitis labrusca) }\end{array}$ & $\mathrm{R}$ & 3,5-diglucosidic anthocyanins & $\begin{array}{l}\text { The presence in the } \\
\text { hybrid grape wine }\end{array}$ & HPLC-DAD-ESI-MS ${ }^{n}$ & - & [81] \\
\hline $\begin{array}{c}\text { Hybrid grape Rondo } \\
(\text { Vitis amurensis } \times \text { Vitis vinifera })\end{array}$ & $\mathrm{R}$ & $\begin{array}{l}\text { 3,5-diglucosidic anthocyanins, } \\
\text { gallic acid, trans-piceid and } \\
\text { cis-piceid }\end{array}$ & $\begin{array}{l}\text { High concentrations } \\
\text { in Rondo }\end{array}$ & UPLC-PDA-MS/MS & HCA & [5] \\
\hline Spine grape (Vitis davidii Foex) & $\mathrm{R}, \mathrm{W}$ & $\begin{array}{c}\text { Malvidin-3,5-diglucoside, } \\
\text { syringetin-3-O-glucoside, } \\
\text { dihydroquercetin-3-hexoside and } \\
\text { coutaric acid }\end{array}$ & $\begin{array}{l}\text { High concentrations } \\
\text { in Spine grapes wine }\end{array}$ & HPLC-DAD/ESI-MS & $\begin{array}{l}\text { ANOVA, Duncan's } \\
\text { multiple range tests }\end{array}$ & [82] \\
\hline Vitis amurensis, Vitis davidii & $\mathrm{R}$ & $\begin{array}{l}\text { 3,5-diglucosidic anthocyanins, } \\
\text { phenolic acids, } \\
\text { kaempferol-3-O-glucoside, } \\
\text { quercetin-3-O-rhamnoside }\end{array}$ & $\begin{array}{l}\text { Higher concentrations } \\
\text { in Vitis davidii }\end{array}$ & HPLC-QqQ-MS/MS & PCA & [83] \\
\hline $\begin{array}{c}\text { Hybrid grapes: Baco }(V \text {. Vinifera } \\
\times \text { V. Labrusca } \times \text { V. Riparia } \times \\
\text { V. Rupestris } \times \text { V. aestivalis }), \text { Seybel } \\
(\text { V. Vinifera } \times \text { V. Rupestris } \times \\
\text { V. lincecu }), \text { Clinton }(\text { V. Labrusca } \times \\
\text { V. riparia), Jacquez }(V . \text { Aestivalis } \times \\
\text { V. Cinerea } \times \text { V. Vinifera), Othello } \\
(\text { V. Labrusca } \times \text { V. Riparia } \times \\
\text { V. Vinifera })\end{array}$ & $\mathrm{R}$ & $\begin{array}{l}\text { Proportion of mono-glucoside } \\
\text { and acetylated anthocyanins }\end{array}$ & $\begin{array}{l}\text { The ratio lower than } \\
\text { three indicates hybrid } \\
\text { grape wine }\end{array}$ & LC-MS-MS & - & [84] \\
\hline $\begin{array}{c}\text { Beihong (Vitis vinifera } \times \\
\text { Vitis amurensis) }\end{array}$ & $\mathrm{R}$ & Mainly phenolic and amino acids & Specific fingerprints & NMR & PCA & [85] \\
\hline
\end{tabular}




\subsection{Geographical Origin and Phenolic Compounds}

The mineral content and the stable isotope ratios (that are present in soil) are a function of geographical origin of wines and are able to describe plant growth and development, environmental contamination, and geological factors [86]. Nevertheless, also phenolic compounds were applied for the evaluation of the geographical origin; in fact, the composition of phenolic compounds in wine does not only depend on the grape variety, but it is also influenced by the viticulture practices, environmental conditions, and winemaking technologies $[87,88]$. In addition, the origin is an important factor for the consumer's choice, as well as for the protection of the reputation and value of products that are recognized by the European Union under PDO (Protected Designation of Origin) and PGI (Protected Geographical Indication) classifications [15,89].

Some studies were done on the evaluation of the phenolic content (hydroxybenzoic and hydroxycinnamic acids, flavan-3-ols and stilbenes) of Riesling wines from the Czech Republic $[8,13,87]$. Using a CVA, significant markers of the geographical origin of Riesling wines proved to be $p$-coutaric acid, (+)-catechin, (-)-epicatechin, trans-resveratrol, and cis-resveratrol (Table 3). To emphasize their suitability as markers for the geographical origin, different vintages of Riesling wines were examined, and the analysis showed that these phenolic compounds were not influenced by the vintage year [8]. As already mentioned above, another study of white wines made from European and interspecific grape varieties from Czech Republic highlighted the importance of hydroxycinnamates (caftaric acid), flavan-3-ols ((+)-catechin, (-)-epicatechin), and stilbenes (cis-piceid), in addition to hydroxybenzoic acids (protocatechuic acid and $p$-hydroxybenzoic acid) for the differentiation of their geographical origin [40]. Phenolic acids were shown to be important markers for the separation of Chardonnay, Feteasca Regala, Pinot Gris, Riesling, and Sauvignon Blanc wines from different geographical regions of France and Romania [43,69-71] and for Australian Chardonnay and Cabernet Sauvignon wines [88]. Interestingly, the differentiation among wines that came from the same country was not possible using a surface-enhanced Raman spectroscopy (SERS) [69], but it was achieved with a FT-Raman [70] having the same samples (as in the study with SERS) and proved to be a sufficient method for other white wine analysis [43]. In the case of Chardonnay wines from Australia, France, Israel, and various regions of Italy, flavonoids appeared to show the highest discrimination potential [89]. Furthermore, using these chemical markers, it was possible to separate wines according to their aging technique: barrels vs. stainless steel tanks [71]. Recently, a comprehensive metabolomic workflow has been applied to discriminate the geographical origin of several Italian monovarietal red wines in their different terroirs [57]. Several putative biomarkers of origin were identified (e.g., flavan-3-ols for Aglianico, Sangiovese, Nerello, and Nebbiolo, flavonols for Sangiovese, and hydroxycinnamates for Cannonau.

There is a lack of scientific literature regarding the geographical authenticity of rosé wines. Lambert et al. [90] have done an extensive study of the quantification of 152 phenolic compounds in rosé wines from different European countries. This research was focused on the establishment of typologies of worldwide rosé wines. However, statistically targeted studies are needed to check which of the phenolic compounds are the most suitable as chemical markers.

Several phenolic compounds were found to represent well the terroir conditions of red wines. For example, flavonols were applied for various wines from France and Spain [28], phenolic acids and flavan-3-ols were applied for Cabernet Sauvignon, Cabernet Franc, Carménère, Merlot, and Syrah cultivated in China [12,66], catechin and quercetin were applied for Cabernet Sauvignon wines from Balkan regions [91] and catechin was applied for Syrah wines from Brazil [11]. However, the correct differentiation of wines according to their geographical origin, in all previously mentioned studies, ranged between 73 and $89 \%$. The profile of anthocyanins showed to be a good marker of Merlot wines from neighboring countries in the South America in combination with a support vector machine (SVM) classifier [6]. High accuracy in prediction $(93.73 \%)$ was obtained in the distinction between Brazil versus non-Brazilian wines, while the least accurate model was $79.16 \%$ (for the Merlot from Chile versus non-Chile). Furthermore, anthocyanins were used for Cabernet 
Sauvignon and Merlot wines produced in China and Balkan Peninsula, red wines from Argentina and Czech Republic. The main chemical markers for the authenticity in these studies were malvidin-3-O-glucoside and its derivatives $[14,19,92,93]$ as well as delphinidin-3-O-glucoside and delphinidin-3-O-p-coumarylglucoside [53]. Likewise, anthocyanins measured with the UV-Vis spectroscopy were applied for the recognition of monovarietal and blended wines from different viticulture regions of Spain [94]. Regarding Malbec wines from Argentina, the evaluation of their phenolic profiles showed that the most descriptive compounds of the terroir were caftaric acid, quercetin-3-O-glucoside, and (+)-catechin [15]. Spanish wines Rías Baixas and Ribeira Sacra labeled with Designation of Origin, along with various German, Greek, Croatian wines, were determined using phenolic acids, flavonoids, tannins, and stilbenes, as reported in Table 4 [7,38,45].

\subsection{Winemaking}

All the steps of the winemaking procedure (prefermentation treatments, fermentation/maceration, stabilization, addition of fining agents, corrections, and aging) may impact the quality of wines. The higher fermentation and maceration temperatures increase the content of phenolic compound; the extended maceration time increases the levels of tannins [95].

Concerning maceration and fermentation, there are only some studies based on the associated phenolic quality markers. The phenolic profile of Cava sparkling wine was characterized by Bosch-Fusté et al. and Izquierdo-Llopart et al. [96,97]. The quality parameters were phenolic acids (Table 5). PCA showed a good clustering and separation of monovarietal and polyvarietal wines using syringic, gallic, caffeic, coumaric, and caftaric acids. These analyses confirmed that hydroxycinnamic acids, their esters, and tartaric acid are important markers in sparkling wines. In addition, rosé and Chardonnay cavas had the highest phenolic contents, while the classical blends of Macabeu, Xarel·lo, and Parellada cavas contained low levels [97]. 
Table 4. Phenolics as chemical markers proposed for the geographical origin.

\begin{tabular}{|c|c|c|c|c|c|c|}
\hline Grape Variety & Wine Origin & Chemical Markers & Role of Chemical Markers & $\begin{array}{l}\text { Analytical } \\
\text { Method }\end{array}$ & $\begin{array}{l}\text { Statistical } \\
\text { Method }\end{array}$ & References \\
\hline Riesling & Czech Republic & $\begin{array}{c}\text { Gallic acid, caffeic acid, caftartic } \\
\text { acid, } p \text {-coutaric acid, ferulic acid } \\
\text { ethylester, } p \text {-coumaric acid } \\
\text { ethylester, }(+) \text {-catechin, } \\
(-) \text {-epicatechin }\end{array}$ & $\begin{array}{l}\text { Different absolute concentrations } \\
\text { of phenolics in comparison with } \\
\text { Riesling from other origins from } \\
\text { the literature }\end{array}$ & HPLC-DAD & $\begin{array}{l}\text { ANOVA, } \\
\text { CVA, LSD }\end{array}$ & [87] \\
\hline Riesling & Czech Republic & $\begin{array}{c}\text { Protocatechuic acid, } \\
\text { p-hydroxybenzoic acid, caftaric } \\
\text { acid, } p \text {-coutaric acid, } \\
\text { trans-resveratrol, cis-resveratrol }\end{array}$ & $\begin{array}{l}\text { Specific absolute concentration of } \\
\text { each phenolic through } \\
\text { five regions }\end{array}$ & HPLC-DAD & $\begin{array}{l}\text { ANOVA, } \\
\text { CDA, LSD, } \\
\text { PCA }\end{array}$ & [13] \\
\hline Riesling & Czech Republic & $\begin{array}{c}\text { p-coutaric acid, trans-resveratrol, } \\
\text { cis-resveratrol, }(+) \text {-catechin, } \\
(-) \text {-epicatechin }\end{array}$ & $\begin{array}{l}\text { Specific absolute concentration of } \\
\text { each phenolic through } \\
\text { five regions }\end{array}$ & HPLC-DAD & $\begin{array}{l}\text { ANOVA, } \\
\text { CVA, LSD, } \\
\text { PCA }\end{array}$ & [8] \\
\hline $\begin{array}{c}\text { Aurelius, Chardonnay, } \\
\text { Müller Thurgau, Moravian } \\
\text { Muscat, Hibernal, } \\
\text { Malverina, Merzling }\end{array}$ & Czech Republic & $\begin{array}{c}\text { Protocatechuic acid, } \\
\text { p-hydroxybenzoic acid, caftaric } \\
\text { acid, cis-piceid, (+)-catechin and } \\
(-) \text {-epicatechin }\end{array}$ & $\begin{array}{c}\text { Specific set of absolute } \\
\text { concentrations of each phenolic } \\
\text { through two regions }\end{array}$ & HPLC-DAD & ANOVA & [40] \\
\hline $\begin{array}{c}\text { Chardonnay, Feteasca } \\
\text { Regala, Sauvignon Blanc }\end{array}$ & France and Romania & $\begin{array}{c}\text { Mainly phenolic acids at } 655,703 \\
755,834,973, \text { and } 1601 \mathrm{~cm}^{-1}\end{array}$ & $\begin{array}{l}\text { Different fingerprints } \\
\text { (band intensity) }\end{array}$ & SERS & LDA & [69] \\
\hline $\begin{array}{l}\text { Feteasca Regala, } \\
\text { Sauvignon Blanc }\end{array}$ & France and Romania & $\begin{array}{l}\text { Mainly phenolic acids at }-709, \\
-887,-740,-721,-503, \text { and } \\
-628 \mathrm{~cm}^{-1}\end{array}$ & $\begin{array}{l}\text { Different fingerprints } \\
\text { (band intensity) }\end{array}$ & FT-Raman & SLDA & [70] \\
\hline $\begin{array}{l}\text { Chardonnay, Pinot Gris, } \\
\text { Riesling, Sauvignon }\end{array}$ & France and Romania & $\begin{array}{c}\text { Mainly phenolic acids at }-451 \text {, } \\
1453,-455,503,1407,1428 \text {, and } \\
1457 \mathrm{~cm}^{-1}\end{array}$ & $\begin{array}{l}\text { Different fingerprints } \\
\text { (band intensity) }\end{array}$ & FT-Raman & LDA & [81] \\
\hline $\begin{array}{l}\text { Chardonnay, Pinot Gris, } \\
\text { Riesling, Sauvignon Blanc }\end{array}$ & France and Romania & $\begin{array}{l}\text { Gallic acid, ferulic acid, } \\
\text { cis-caftaric acid, quercitin }\end{array}$ & $\begin{array}{l}\text { Different fingerprints } \\
\text { (peak intensity) }\end{array}$ & NMR & LDA & [43] \\
\hline $\begin{array}{l}\text { Chardonnay, Cabernet } \\
\text { Sauvignon }\end{array}$ & Australia & $\begin{array}{c}\text { Cinnamic acid, tartaric acid, } \\
\text { myricetin }\end{array}$ & $\begin{array}{l}\text { Specific absolute concentrations } \\
\text { (not presented in the article) }\end{array}$ & $\begin{array}{l}\text { HPLC- } \\
\text { DAD/MS }\end{array}$ & LDA, PCA & [89] \\
\hline Chardonnay & $\begin{array}{l}\text { Australia, France, } \\
\text { Israel, Italy }\end{array}$ & $\begin{array}{l}\text { Mainly flavonols, flavan-3-ols, } \\
\text { flavones, flavanones }\end{array}$ & $\begin{array}{l}\text { Specific absolute concentrations } \\
\text { for each region and country }\end{array}$ & $\begin{array}{c}\text { UHPLC- } \\
\text { ESI/QTOF-MS }\end{array}$ & $\begin{array}{l}\text { ANOVA, } \\
\text { OPLS-DA }\end{array}$ & [88] \\
\hline
\end{tabular}


Table 4. Cont

\begin{tabular}{|c|c|c|c|c|c|c|}
\hline Grape Variety & Wine Origin & Chemical Markers & Role of Chemical Markers & $\begin{array}{l}\text { Analytical } \\
\text { Method }\end{array}$ & $\begin{array}{l}\text { Statistical } \\
\text { Method }\end{array}$ & References \\
\hline $\begin{array}{c}\text { Bobal, Cabernet Sauvignon, } \\
\text { Garnacha, Merlot, } \\
\text { Tempranillo }\end{array}$ & France and Spain & Flavonols & $\begin{array}{l}\text { Specific molar percentage of } \\
\text { eight flavonols through } \\
\text { the samples }\end{array}$ & $\begin{array}{l}\text { LC-DAD- } \\
\text { ESI-MSn }^{n}\end{array}$ & $\begin{array}{l}\text { Student- } \\
\text { Newman- } \\
\text { Keuls test }\end{array}$ & [28] \\
\hline $\begin{array}{c}\text { Cabernet Sauvignon, } \\
\text { Cabernet Franc, Carménère } \\
\text { Merlot, Syrah }\end{array}$ & China & Flavan-3-ols and phenolic acids & $\begin{array}{l}\text { Significant differences in the } \\
\text { absolute concentrations }\end{array}$ & $\begin{array}{l}\text { HPLC- } \\
\text { DAD/PDA }\end{array}$ & PCA & [66] \\
\hline Cabernet Sauvignon & China & $\begin{array}{c}\text { Gallic acid, }(+) \text {-catechin, } \\
\text { (-)-epicatechin, procyanidin B1, } \\
\text { procyanidin } \\
\text { B2, procyanidin C1 }\end{array}$ & Specific absolute concentrations & $\begin{array}{l}\text { HPLC-QqQ- } \\
\text { MS/MS }\end{array}$ & $\begin{array}{c}\text { PCA, } \\
\text { PLS-DA, } \\
\text { OPLS-DA }\end{array}$ & [12] \\
\hline Cabernet Sauvignon & $\begin{array}{c}\text { Bulgaria, Croatia, } \\
\text { Macedonia, } \\
\text { Montenegro, Serbia }\end{array}$ & Catechin, quercetin & $\begin{array}{c}\text { Specific absolute concentrations } \\
\text { through the samples from } \\
\text { different countries }\end{array}$ & HPLC-DAD/FL & ANOVA & [91] \\
\hline Syrah & Brazil & Catechin & $\begin{array}{l}\text { Notable differences in absolute } \\
\text { concentrations through } \\
\text { the terroirs }\end{array}$ & HPLC-DAD & $\begin{array}{c}\text { ANOVA, } \\
\text { PCA, } \\
\text { Tukey test }\end{array}$ & [11] \\
\hline Merlot & $\begin{array}{l}\text { Argentina, Brazil, } \\
\text { Chile, Uruguay }\end{array}$ & Anthocyanins & Specific absolute concentration & $\begin{array}{l}\text { HPLC- } \\
\text { DAD/MS }\end{array}$ & SVM & [6] \\
\hline $\begin{array}{c}\text { Cabernet Sauvignon, } \\
\text { Merlot }\end{array}$ & China & $\begin{array}{c}\text { Percentage of } \\
\text { malvidin-3-O-glucoside and its } \\
\text { derivatives to the total content } \\
\text { of anthocyanins }\end{array}$ & $\begin{array}{l}\text { Specific percentage through } \\
\text { the terroirs }\end{array}$ & HPLC-MS/MS & Tukey test & [92] \\
\hline $\begin{array}{l}\text { Teran, Plavac mali, Merlot, } \\
\text { Cabernet Sauvignon }\end{array}$ & Croatia & Anthocyanins & Specific absolute concentrations & $\begin{array}{l}\text { UPLC-QqQ- } \\
\text { MS/MS }\end{array}$ & $\begin{array}{c}\text { ANOVA, } \\
\text { LSA, } \\
\text { SLDA }\end{array}$ & [19] \\
\hline $\begin{array}{l}\text { Aspiran, Bonarda, } \\
\text { Cabernet Sauvignon, } \\
\text { Malbec, Merlot, Sangiovese, } \\
\text { Syrah, Tempranillo }\end{array}$ & Argentina & $\begin{array}{c}\text { Malvidin-3-O-glucoside and } \\
\text { its derivatives }\end{array}$ & $\begin{array}{l}\text { Specific absolute concentrations } \\
\text { through the terroirs (not } \\
\text { presented in the article) }\end{array}$ & HPLC-MS & $\begin{array}{l}\text { MCR-ALS, } \\
\text { D-UPLS }\end{array}$ & [14] \\
\hline
\end{tabular}


Table 4. Cont.

\begin{tabular}{|c|c|c|c|c|c|c|}
\hline Grape Variety & Wine Origin & Chemical Markers & Role of Chemical Markers & $\begin{array}{l}\text { Analytical } \\
\text { Method }\end{array}$ & $\begin{array}{c}\text { Statistical } \\
\text { Method }\end{array}$ & References \\
\hline Cabernet Sauvignon & $\begin{array}{c}\text { Bulgaria, Croatia, } \\
\text { Macedonia, } \\
\text { Montenegro, Serbia }\end{array}$ & Anthocyanins & Specific absolute concentrations & $\begin{array}{l}\text { HPLC-DAD, } \\
\text { DPPH }\end{array}$ & - & [93] \\
\hline $\begin{array}{c}\text { Blaufränkisch, Blauer } \\
\text { Portugieser, Saint Laurent }\end{array}$ & Czech Republic & $\begin{array}{c}\text { Delphinidin-3-O-glucoside, } \\
\text { malvidin-3-O-glucoside, } \\
\text { delphinidin-3-O-p- } \\
\text { coumarylglucoside }\end{array}$ & $\begin{array}{l}\text { Different absolute concentrations } \\
\text { of anthocyanins in comparison } \\
\text { with studied wines from other } \\
\text { origins from the literature }\end{array}$ & HPLC-DAD & $\begin{array}{l}\text { ANOVA, } \\
\text { CDA, LSD, } \\
\text { PCA }\end{array}$ & [53] \\
\hline $\begin{array}{l}\text { Various monovarietal and } \\
\text { blended wines }\end{array}$ & Spain & $\begin{array}{l}\text { Hydroxycinnamic acids (for } \\
\text { white wines), anthocyanin (for } \\
\text { red wines) }\end{array}$ & Not specified & UV-Vis & SVM & [94] \\
\hline Malbec & Argentina & $\begin{array}{c}\text { (+)-Catechin, caftaric acid and } \\
\text { quercetin-3-O-glucoside }\end{array}$ & $\begin{array}{c}\text { Specific absolute concentrations } \\
\text { through six regions (lowest } \\
\text { content was in Rivadavia) }\end{array}$ & HPLC-DAD & DA & [15] \\
\hline Rías Baixas, Ribeira Sacra & Spain & $\begin{array}{l}\text { Syringic acid, malvin, oenin, } \\
\text { (+)-catechin, (-)-epicatechin, } \\
\text { quercetin, trans-resveratrol }\end{array}$ & $\begin{array}{l}\text { Different absolute concentrations } \\
\text { (not presented in the article) }\end{array}$ & HPLC-DAD & $\begin{array}{l}\text { LDA, PCA, } \\
\text { SIMCA, } \\
\text { SVM }\end{array}$ & [35] \\
\hline $\begin{array}{l}\text { Lemberger, Pinot Blanc, } \\
\text { Pinot Gris, Müller-Thurgau, } \\
\text { Riesling, Gewürztraminer, } \\
\text { Pinot Noir }\end{array}$ & Germany & Phenolic and amino acids & Specific fingerprint & NMR & $\begin{array}{l}\text { CV, LDA, } \\
\text { MANOVA, } \\
\text { MC, NCM, } \\
\text { PCA, }\end{array}$ & [43] \\
\hline $\begin{array}{l}\text { Moschofilero, Asyrtiko, } \\
\text { Agiorgitiko, Mandilaria, }\end{array}$ & Greece & $\begin{array}{l}\text { Gallic acid, trans-caffeic, } \\
\text { (-)-epicatechin }\end{array}$ & $\begin{array}{c}\text { Both } \mathrm{W} \text { and } \mathrm{R} \text { wines from } \\
\text { Santorini had twice more of gallic } \\
\text { acid and lower concentrations of } \\
\text { trans-caffeic, (-)-epicatechin than } \\
\text { wines from Nemea }\end{array}$ & NMR & $t$ test & [7] \\
\hline
\end{tabular}

CDA—categorical data analysis; DPPH—2,2,1-diphenyl-1-picrylhydrazyl radical scavenging; ESI—electrospray ionization;. FL—fluorescence. 
Table 5. Chemical markers for the winemaking quality control.

\begin{tabular}{|c|c|c|c|c|c|c|}
\hline Grape Variety & Winemaking Method & Chemical Markers & Role of Chemical Markers & Analytical Method & Statistical Method & References \\
\hline Not reported & Cava sparkling wine & $\begin{array}{c}\text { Phenolics with absorbance } \\
\text { at } 280 \mathrm{~nm}\end{array}$ & $\begin{array}{c}\text { Decrease of } \\
\text { hydroxycinnamic acids in } \\
\text { sparkling wine }\end{array}$ & HPLC-DAD/ESI-TOFMS & MANOVA & [96] \\
\hline $\begin{array}{c}\text { Chardonnay (Cd), } \\
\text { Macabeu, Xarel·lo, } \\
\text { Parellada, Pinot Noir } \\
\text { (PN), Garnacha (Gn), } \\
\text { Trepat (Tp) }\end{array}$ & Cava sparkling wine & Phenolic acids & $\begin{array}{l}\text { High amounts Cd, PN, Gn } \\
\text { and Tp low—in Macabeu, } \\
\text { Xarel-lo and Parellada }\end{array}$ & HPLC-DAD & PCA & [97] \\
\hline Pinot Blanc & $\begin{array}{l}\text { Prefermentative cold } \\
\text { maceration with } \\
\text { pectolytic enzyme }\end{array}$ & $\begin{array}{l}\text { Trans-caftaric } \\
\text { acid and astilbin }\end{array}$ & $\begin{array}{l}\text { Higher concentrations in } \\
\text { wines with cold maceration }\end{array}$ & $\begin{array}{l}\text { HPLC-DAD/FLD, } \\
\text { HPLC-MS }\end{array}$ & PCA & [98] \\
\hline Primitivo & $\begin{array}{c}\text { Destemmed } 100 \%, 75 \%, \\
50 \% \text { of grapes cluster and } \\
\text { stem contact for all time } \\
\text { fermentation }\end{array}$ & Anthocyanins & $\begin{array}{l}\text { Concentration increasing } \\
\text { when less stems are used }\end{array}$ & HPLC-DAD & $\begin{array}{c}\text { ANOVA, } \\
\text { Duncan multiple } \\
\text { comparison test, PCA }\end{array}$ & [99] \\
\hline Merlot, Syrah, Tannat & $\begin{array}{l}\text { Traditional maceration; } \\
\text { addition of maceration } \\
\text { enzymes; cold soak }\end{array}$ & Anthocyanins & $\begin{array}{l}\text { Higher concentration } \\
\text { during cold soak }\end{array}$ & HPLC-DAD & Tukey test & [50] \\
\hline Pinot Noir & $\begin{array}{l}\text { Thermal maceration; } \\
\text { treatment after a stuck } \\
\text { fermentation; } \\
\text { fermentation with } 20 \% \\
\text { whole grape clusters; } \\
100 \% \text { raisin grapes }\end{array}$ & $\begin{array}{l}\text { Ratios of cyclic and non-cyclic } \\
\text { proanthocyanidins }\end{array}$ & $\begin{array}{l}\text { Higher concentration of } \\
\text { proanthocyanidins with } \\
\text { raisin grapes }\end{array}$ & HPLC-HRMS/MS & PCA & [100] \\
\hline $\begin{array}{l}\text { Cabernet Sauvignon, } \\
\text { Merlot, Monastrell }\end{array}$ & Wine blending & $\begin{array}{l}\text { Groups of phenolics at } 520 \mathrm{~nm} \\
\text { and } 620 \mathrm{~nm}, \\
\text { petunidin-3-O-glucoside and } \\
\text { peonidin-3-O-glucoside }\end{array}$ & $\begin{array}{l}\text { Specific fingerprints } \\
\text { through different blends }\end{array}$ & $\begin{array}{c}\text { HPLC-DAD, } \\
\text { UV-Vis spectrometry }\end{array}$ & DA & [101] \\
\hline
\end{tabular}


Dupas de Matos et al. [98] presented differences in the phenolic composition of Pinot Blanc wines made with and without prefermentative cold maceration in the presence of pectolytic enzymes. The samples from both winemaking procedures were best described by the concentrations of trans-caftaric acid and astilbin.

Suriano et al. [99] investigated the maceration held with different amounts of destemmed Primitivo grapes. Anthocyanins were the compounds that were most differentiating between the maceration without and with stems. Primitivo wines made with destemmed grapes had higher concentrations of monomeric and total anthocyanins both at racking and after 12 months storage compared to the wines with $25 \%$ and $50 \%$ grapes with stems that were richer in tannins. Anthocyanins were used as chemical markers of three types of maceration of Merlot, Syrah, and Tannat [50]. Using the relative anthocyanin profile, it was possible to cluster the samples according to the winemaking technique. In addition, the content of anthocyanins increased during cold soaking maceration in Merlot and Tannat wines. Merkyte et al. [100] compared different winemaking processes of Pinot Noir wines and reported that cyclic and non-cyclic proanthocyanidins and their relative ratio showed to be affected by the use of raisin grapes for winemaking and by the occurrence of stuck fermentations. The ratios between cyclic proanthocyanidins and total proanthocyanidins (with the same number of monomers) were the highest in wine that experienced stuck fermentation in comparison with other samples, possibly because they may be extracted in the first steps of maceration. The wine from raisin grapes showed the lowest ratios, meaning that it contained higher concentrations of cyclic proanthocyanidins among all the samples.

Red wine blending was studied by Lorenzo et al. [101], who evaluated the differentiation of wines according to the aging time and the type of wine using discriminant analysis, showing that the main markers were anthocyanins and their derivatives.

Cejudo-Bastante et al. [22] studied the phenolic profile of different stages of vinification in grapes skins, musts, and wine in Tempranillo and minority grape varieties of Castilla-La Mancha region (Spain), showing that rosé wines with the highest amount of flavonols were Tortosí and Bobal wines, while Moravia Dulce and Moravia Agria wines showed low quantities. The highest values of anthocyanins were observed in Bobal and Moravia Agria wines (in the middle and at the end of alcoholic fermentation). However, their content decreased after malolactic fermentation. Interestingly, Tortosí and Moravia Dulce wines showed increment of anthocyanins after malolactic fermentation. In addition, wines made from Rojal grapes were practically absent both of flavonols and anthocyanins. Another qualitative study was done by Loizzo et al. [102] that was focused on an investigation of Passito wines made from non-macerated white grapes: Guarnaccia, Malvasia, and Moscato. Phenolic compounds found in the highest concentrations were gallic and caftaric acids, (+)-catechin and oligomeric procyanidins (due to the polymerization of simple flavonoids).

\subsection{Aging}

During the wine aging in wooden barrels, phenolics migrate from wood to wine and cause changes in color and mouthfeel sensations. Moreover, the micro-oxygenation through the pores of wood is influencing the color of red wines (decrement of free anthocyanins and formation of polymeric pigments). The profile of phenolic compounds in the aged wines depends on many factors, such as type of barrel wood, environmental humidity, toasting degree, wine alcohol content, aging time, wood structure, and its polyphenolic load. The typical taste of the wines stored in wood is one of the quality parameters [32,103]. The chemical changes of wine during aging can be observed in bottles. The study by Arapitsas et al. [104] was focused on the interactions between $\mathrm{SO}_{2}$ and phenolics, as well as amino acids in wines produced from 1986 until 2016. It showed that the best discriminants for white wines were sulfonated indoles, and for red wines, they were sulfonated monomeric and oligomeric flavan-3-ols.

A few studies suggested that phenolic acids diffusing from wood can be used as chemical markers for the wines aged in wooden barrels (Table 6). Matejicek et al. [103] found that the content of ellagic acid showed a noticeable difference between wines that were aged in the medium and 
highly toasted barrels; thus, ellagic acid can be used as the marker of maturity of oak barrique wines. However, the degree of toasting in each toasting category did not affect its concentration. Another investigation by Sanz et al. [105] concerning phenolic acids showed that 2,4-dihydroxybenzoic acid together with certain flavonoids (e.g., robinetin, fustin, butin, tetrahydroxydihydroflavonol, and trihydroxymethoxydihydroflavonol) were a good set of discriminators of wines aged in acacia wood, since these compounds were not present in wines stored in oak wood. Alañón et al. [106] showed that the profiles of benzoic acids (protocatechuic, gallic, and ellagic acid) and monomeric anthocyanins were the marker for the wine aged in chestnut wood. The content of protocatechuic acid was different in wine made in non-toasted and toasted barrels, but its concentration was not changing during the aging (in 3 and 6 months), while the content of gallic and ellagic acids were discriminants of wine aging. The monomeric anthocyanins described the best changes during aging in non-toasted chestnut barrels. In addition, the lower concentration of total flavonols in wines that were treated with chestnut chips than the samples in barrels might be a possible marker for this type of wine aging. Chinnici et al. [107] showed that flavonoids such as eryodictiol, sakuranetin, pinocembrin, and chrysin appeared to be the most distinctive phenolic compounds of wine stored in cherry wood. These compounds were not found in samples aged in oak barrels or steel tanks.

In the studies of wine aging comparing the storage in oak barrels versus the addition of oak chips [108,109], protocatechuic and vanillic acids, together with (-)-epicatechin and anthocyanins were the most discriminant markers. Del Alamo et al. [108] investigated the red wine aging in different oak wood (American, French, and Hungarian) systems: barrels, chips, and staves. They indicated the set of chemical markers; moreover, they also analyzed the same samples after 2 years of storage in bottle and found that differences between these three systems grew during the bottling period. Ortega-Heras et al. [109] showed that wines treated with chips are higher or lower in phenolic content than wines aged in barrels depending on their variety and vintage. Baiano et al. [110] investigated Aglianico and Montepulciano wines treated with and without oak chips; the usage of oak chips determined an increase of polymerized anthocyanins and tannins in Aglianico and only polymerized anthocyanins in Montepulciano wines. After one year of storage in bottles, the decrease in monomeric anthocyanins concentration was higher in samples treated with oak chips in both type of wines. Finally, extensive studies of wine aging in barrels made from different type of wood (French oak, American oak, acacia, and chestnut) were carried out by Basalekou et al. [32,111]. The first studies suggested that the Fourier-transform infrared spectroscopy (FT-IR) spectral regions from 1800 to $1500 \mathrm{~cm}^{-1}$ and from 1300 to $900 \mathrm{~cm}^{-1}$ showed a good discrimination of differently aged white and red wines. However, the correlation based on their aging time was poor. In the later publication, they focused on the content of ellagitannins. The levels of ellagitannins depended on the type of wood, its geographic origin, and the wine aging time. The wines aged in chestnut wood had the highest concentration of ellagic acid, whereas French oak appeared to enhance the content of ellagic acid more than American oak. The lowest concentration of ellagic acid was observed in wines treated with chips. In agreement with previous observation, ellagic acid was absent in samples aged in acacia wood. Furthermore, the application of ellagitannins as quality markers could assist in the industry, since they can be used for both white and red wines with the same methodology. 
Table 6. Phenolics as chemical markers for aging in wood.

\begin{tabular}{|c|c|c|c|c|c|c|c|}
\hline Grape Variety & Aging Time & Aging Method & Chemical Markers & Role of Chemical Markers & Analytical Method & Statistical Method & References \\
\hline $\begin{array}{l}\text { Chardonnay, Pinot } \\
\text { Gris, Verdicchio, } \\
\text { Amarone, } \\
\text { Sagrantino, } \\
\text { Sangiovese, Tannat }\end{array}$ & 1986-2016 & In bottles & $\begin{array}{l}\text { Sulfonated indoles for } \\
\text { white wines, sulfonated, } \\
\text { monomeric, and } \\
\text { oligomeric flavan-3-ols } \\
\text { for red wines }\end{array}$ & $\begin{array}{c}\text { Specific absolute } \\
\text { concentrations through } \\
\text { different varieties and } \\
\text { vintages }\end{array}$ & UHPLC-MS/MS & $\begin{array}{c}\text { One-way ANOVA, } \\
\text { Tukey test }\end{array}$ & [104] \\
\hline $\begin{array}{c}\text { Blend of Cabernet } \\
\text { Sauvignon and } \\
\text { Merlot }\end{array}$ & 200 days & $\begin{array}{l}\text { Medium toasted and } \\
\text { highly toasted barrels } \\
\text { from oak }\end{array}$ & Ellagic acid & $\begin{array}{l}\text { Higher concentration in } \\
\text { wine from oak barrels }\end{array}$ & HPLC-DAD & - & [103] \\
\hline Syrah & $\begin{array}{l}2,4,6,9 \text {, and } \\
12 \text { months }\end{array}$ & $\begin{array}{l}\text { Toasted acacia and } \\
\text { French oak barrels }\end{array}$ & $\begin{array}{l}\text { 2,4-dihydroxybenzoic } \\
\text { acid and flavonoids }\end{array}$ & $\begin{array}{l}\text { Present only in wine aged in } \\
\text { acacia wood }\end{array}$ & LC-DAD/ESI-MS & - & [105] \\
\hline Tempranillo & $\begin{array}{l}3 \text { and } 6 \text { months in } \\
\text { barrels; } 25 \text { days } \\
\text { with chips }\end{array}$ & $\begin{array}{l}\text { Non-toasted and in } \\
\text { medium toasted } \\
\text { chestnut barrels and } \\
\text { chestnut chips }\end{array}$ & $\begin{array}{l}\text { Benzoic acids, } \\
\text { anthocyanins }\end{array}$ & $\begin{array}{l}\text { Specific absolute } \\
\text { concentrations of increasing } \\
\text { on the aging and } \\
\text { distributing differently due } \\
\text { to aging method }\end{array}$ & LC-DAD/ESI-MS & PCA & [106] \\
\hline $\begin{array}{c}\text { Blend of } \\
\text { Sangiovese and } \\
\text { Merlot }\end{array}$ & 4 months & $\begin{array}{l}\text { Oak and cherry wood } \\
\text { barriques, steel tanks }\end{array}$ & Flavanones & $\begin{array}{l}\text { Present only in cherry } \\
\text { barriques }\end{array}$ & HPLC-DAD & $\begin{array}{r}\text { Post-hoc, } \\
\text { LSD, PCA }\end{array}$ & [107] \\
\hline Tinta del País & 6 and 12 months & $\begin{array}{c}\text { Traditional barrels, oak } \\
\text { chips and oak staves } \\
\text { (American, French and } \\
\text { Hungarian), } \\
\text { stainless-steel tanks }\end{array}$ & $\begin{array}{c}\text { Epicatechin, phenolic } \\
\text { acids, } \\
\text { anthocyanins }\end{array}$ & $\begin{array}{c}\text { Specific absolute } \\
\text { concentrations of phenolics } \\
\text { influenced by aging time, } \\
\text { type and wood }\end{array}$ & HPLC-DAD & LDA, PCA & [108] \\
\hline $\begin{array}{l}\text { Mencia, Tinta } \\
\text { del País }\end{array}$ & $\begin{array}{l}3,6,9 \text {, and } \\
12 \text { months }\end{array}$ & $\begin{array}{l}\text { Oak barrels and oak } \\
\text { chips (American and } \\
\text { French) }\end{array}$ & Anthocyanins & $\begin{array}{l}\text { Specific set of absolute } \\
\text { concentrations (not } \\
\text { presented in the article) }\end{array}$ & HPLC & DA & [110] \\
\hline $\begin{array}{c}\text { Aglianico, } \\
\text { Montepulciano }\end{array}$ & 12 months & $\begin{array}{c}\text { With and without oak } \\
\text { chips }\end{array}$ & $\begin{array}{c}\text { Anthocyanins, } \\
\text { tannins }\end{array}$ & $\begin{array}{l}\text { Polymerization of these } \\
\text { markers when oak chips } \\
\text { are used }\end{array}$ & $\begin{array}{l}\text { HPLC-DAD/ } \\
\text { ESI-MS/MS }\end{array}$ & $t$ test, PCA & [110] \\
\hline $\begin{array}{l}\text { Vilana, Dafni, } \\
\text { Kotsifali, } \\
\text { Mandilari }\end{array}$ & $\begin{array}{l}3,6,9 \text {, and } \\
12 \text { months }\end{array}$ & $\begin{array}{l}\text { Medium toasted barrels } \\
\text { (French oak, American } \\
\text { oak, acacia and } \\
\text { chestnut), stainless steel }\end{array}$ & $\begin{array}{l}\text { Spectral regions from } \\
1800 \text { to } 1500 \mathrm{~cm}^{-1} \text { and } \\
\text { from } 1300 \text { to } 900 \mathrm{~cm}^{-1}\end{array}$ & $\begin{array}{l}\text { Different fingerprints } \\
\text { according to aging time } \\
\text { and type }\end{array}$ & FT-IR & LDA & [111] \\
\hline $\begin{array}{l}\text { Vilana, Dafni, } \\
\text { Kotsifali, } \\
\text { Mandilari }\end{array}$ & 3,6 , and 9 months & $\begin{array}{l}\text { Tanks with oak sticks } \\
\text { and barrels (French oak, } \\
\text { American oak, acacia, } \\
\text { and chestnut) }\end{array}$ & Ellagitannins & $\begin{array}{c}\text { Content decrement: } \\
\text { chestnut }>\text { French oak }> \\
\text { American oak }>\text { chips }> \\
\text { acacia }\end{array}$ & FT-IR & PLS & [32] \\
\hline
\end{tabular}




\subsection{Vintage Year}

The vintage year is an important quality parameter, that strongly influences the price of wine, and it is mainly affected by various environmental factors such as the viticultural practice and meteorological (climatic) conditions. During the wine aging in bottle, the phenolic composition changes and affects the wine color, astringency, and bitterness perceptions [17,112].

Some studies $[7,44,45]$ investigated the phenolic profile of white wines over two different vintages using the NMR technique. PCA and partial least squares-discriminant analysis (PLS-DA) of the NMR spectra showed a clear differentiation between the samples, using mainly phenolic acids as variables. Anastasiadi et al. [7] demonstrated that the vintage is related to the environmental conditions, since both white and red wines made in 2005 were characterized by lower concentrations of polyphenols, which can be explained by the overhydration of grape berries due to heavy rainfalls that year in Nemea (Greece). Studies [43,69-71] using SERS and FT-Raman analysis showed the application of phenolic acids for white wine made with grapes from different harvest years. In the studies of 2018 [69,70], a weaker classification (by linear discriminant analysis (LDA)) was obtained using SERS (83.3\%) in comparison to FT-Raman (96.9\%), which proved to be better analysis technique for the vintage differentiation, since SERS does not provided signals for specific phenolic acids that are important discriminators.

Other studies based on vintage analysis were made with red deep-colored wines and are presented in Table 7. In Pinotage wines, concentrations of caffeic acid were stable throughout the aging process, while levels of malvidin-3-O-glucoside were decreasing. It was suggested that the increment of the ratio between caffeic acid and malvidin-3-O-glucoside reveals the older vintages of Pinotage, as well as the formation of pinotin A [113]. In Tempranillo wines produced in 2000, the percentages of delphinidin-3-O-glucoside, petunidin-3-O-glucoside, and malvidin-3-O- $p$-coumarylglucoside were higher, and the content of malvidin-3-O-glucoside was lower than in 2001 and 2002 due to climatic factors linked to the year of production [114]. The study on Sangiovese wines showed the decrement of the absolute concentration of simple glucosides of anthocyanins and vitisin B pigments in the older wines, whereas pinotin A pigments were increasing through the years [115]. In the work of Chira et al. [17], the profile of anthocyanins was in combination with tannins and sensory data. However, the most relevant marker for the vintage year differentiation in both Cabernet Sauvignon and Merlot wines was the mean degree of polymerisation of proanthocyanidins. The study by Eder et al. [116] confirmed that the content of total monomeric anthocyanins and malvidin-3-O-glucoside are suitable markers to assess the vintage year. The concentrations of monomeric anthocyanins were reduced by more than $50 \%$ after two years, while pyranoanthocyanins (vitisin $\mathrm{A}, \mathrm{B}$, and pinotin $\mathrm{A}$ ) were independent from the wine age. Monomeric and dimeric flavan-3-ols, proanthocyanidins, and total phenolic content were perfect differentiators of Cabernet Franc, Merlot, Sangiovese, and Syrah wines produced in 2006 and 2007 [65]. The concentrations of catechin, proanthocyanidins, total monomers, and total dimers were significantly different in both Cabernet Franc and Merlot wines from both vintages. The content of these compounds was higher in wines from 2006. Conversely, Sangiovese from vintage 2007 had lower concentrations of catechin, epicatechin, total monomers, and total dimers compared to wines produced during the vintage 2006. The same trend was in Syrah wines, except for the total dimers. 
Table 7. Chemical markers proposed to determine the vintage year.

\begin{tabular}{|c|c|c|c|c|c|c|}
\hline Grape Variety & Vintage & Chemical Markers & Role of Chemical Markers & Analytical Method & Statistical Method & References \\
\hline $\begin{array}{l}\text { Müller-Thurgau, } \\
\text { Riesling }\end{array}$ & 2006,2007 & Amino, organic and phenolic acids & $\begin{array}{c}\text { High concentration of } \\
\text { different acids through } \\
\text { vintages }\end{array}$ & NMR & PLS & [44] \\
\hline $\begin{array}{c}\text { Moschofilero, } \\
\text { Asyrtiko, Agiorgitiko, } \\
\text { Mandilaria, }\end{array}$ & 2005,2006 & $\begin{array}{l}\text { Gallic acid, trans-caffeic acid, } p \text {-coumaric } \\
\text { acid, syringic acid, ferulic acid, (+)-catechin, } \\
\text { (-)-epicatechin, quercetin, kaempferol, } \\
\text { trans-resveratrol }\end{array}$ & $\begin{array}{l}\text { Lower concentration of } \\
\text { polyphenols of samples } \\
\text { from } 2005\end{array}$ & NMR & $t$ test & [7] \\
\hline $\begin{array}{l}\text { Lemberger, Pinot } \\
\text { Blanc, Pinot Gris, } \\
\text { Müller-Thurgau, } \\
\text { Riesling, } \\
\text { Gewürztraminer, } \\
\text { Pinot Noir }\end{array}$ & 2008,2009 & Phenolic and amino acids & $\begin{array}{l}\text { Individual fingerprint } \\
\text { of samples }\end{array}$ & NMR & $\begin{array}{l}\text { CV, LDA, MANOVA, } \\
\text { MC, NCM, PCA, }\end{array}$ & [45] \\
\hline $\begin{array}{c}\text { Chardonnay, Feteasca } \\
\text { Regala, Sauvignon } \\
\text { Blanc }\end{array}$ & 2011-2015 & Malic and tartaric acids & $\begin{array}{l}\text { Different fingerprints } \\
\text { (band intensity) }\end{array}$ & SERS & LDA & [69] \\
\hline $\begin{array}{r}\text { Feteasca Regala, } \\
\text { Sauvignon Blanc }\end{array}$ & 2011-2015 & $\begin{array}{l}\text { Mainly phenolic acids at }-767,-543,-530 \\
-653,1608 \text { and }-881 \mathrm{~cm}^{-1}\end{array}$ & $\begin{array}{l}\text { Different fingerprints } \\
\text { (band intensity) }\end{array}$ & FT-Raman & SLDA & [70] \\
\hline $\begin{array}{l}\text { Chardonnay, Pinot } \\
\text { Gris, Riesling, } \\
\text { Sauvignon }\end{array}$ & 2012-2016 & Caffeic, caftaric, ferulic acids & $\begin{array}{l}\text { Different fingerprints } \\
\text { (band intensity) }\end{array}$ & FT-Raman & LDA & [71] \\
\hline Pinotage & 1996-2002 & $\begin{array}{l}\text { Caffeic acid, malvidin-3-O-glucoside } \\
\text { (MvGl), pinotin A }\end{array}$ & $\begin{array}{l}\text { Increased ratio of caffeic } \\
\text { acid/MvGl through time } \\
\text { and pinotin A content }\end{array}$ & HPLC-MD & $\mathrm{SD}$ & [113] \\
\hline Tempranillo & 2000-2002 & $\begin{array}{c}\text { Delphinidin-3-O-glucoside (DpGl), } \\
\text { petunidin-3-O-glucoside (PtGl), } \\
\text { glucoside, } \\
\text { malvidin-3-O-p-coumarylglucoside } \\
\text { (MvGlCm), malvidin-3-O-glucoside (MvGl) }\end{array}$ & $\begin{array}{c}\text { Increment of DpGl, PtGl } \\
\text { and malvidin- MvGlCm } \\
\text { and decrement of MvGl } \\
\text { through time }\end{array}$ & HPLC & ANOVA, HCA, PCA & [114] \\
\hline Sangiovese & 2008-2010 & Anthocyanins & $\begin{array}{l}\text { Decrement of anthocyanins } \\
\text { and increment of pinotin A } \\
\text { through time }\end{array}$ & UHPLC-DAD-MS/MS & PLS & [115] \\
\hline $\begin{array}{l}\text { Cabernet Sauvignon } \\
\text { (CS), Merlot (M) }\end{array}$ & $\begin{array}{l}\text { Range 1978-2005 (for } \\
\text { CS) and 1979-2003 } \\
\text { (for M) }\end{array}$ & Anthocyanins, tannins & $\begin{array}{l}\text { The specific sum of } \\
\text { concentrations of phenolic } \\
\text { classes through the samples }\end{array}$ & HPLC-DAD & ANOVA, PCA & [17] \\
\hline
\end{tabular}


Table 7. Cont.

\begin{tabular}{|c|c|c|c|c|c|c|}
\hline Grape Variety & Vintage & Chemical Markers & Role of Chemical Markers & Analytical Method & Statistical Method & References \\
\hline Varietal red wines & 2000-2010 & $\begin{array}{c}\text { Monomeric anthocyanins, } \\
\text { malvidin-3-O-glucoside; } \\
\text { pyranoanthocyanins are not effective }\end{array}$ & $\begin{array}{l}\text { Decrement of monomeric } \\
\text { anthocyanins }\end{array}$ & LC-ESI-MS & t-test, PCA & [116] \\
\hline $\begin{array}{l}\text { Cabernet Franc }(\mathrm{CF}), \\
\text { Merlot }(\mathrm{M}), \\
\text { Sangiovese }(\mathrm{Sg}) \\
\text { Syrah }(\mathrm{Sr})\end{array}$ & 2006, 2007 & Flavan-3-ols, tannins & $\begin{array}{c}\text { Lower or higher } \\
\text { concentrations } \\
(2006<2007 \text { in CF, M; } 2006 \\
>2007 \text { in Sg, Sr })\end{array}$ & HPLC-DAD-MS & $\begin{array}{c}\text { ANOVA, PCA, Tukey } \\
\text { test }\end{array}$ & [65] \\
\hline Cabernet Sauvignon & 1971-2003 & Phenolic acids, flavonoids and resveratrol & $\begin{array}{l}\text { Decrement and increment } \\
\text { of concentrations of specific } \\
\text { phenolics during the } \\
\text { timeline }\end{array}$ & HPLC-MS & PCA, PLSR & [112] \\
\hline Cabernet Sauvignon & 2003-2015 & $\begin{array}{l}\text { (+)-catechin, }(-) \text {-epicatechin, } \\
\text { malvidin-3-O-glucoside, malvidin- } \\
\text { 3-O-acetylglucoside }\end{array}$ & $\begin{array}{l}\text { Specific set of absolute } \\
\text { concentrations (not } \\
\text { presented in the article) }\end{array}$ & HPLC-QqQ-MS/MS & $\begin{array}{l}\text { PCA, PLS-DA, } \\
\text { OPLS-DA }\end{array}$ & [12] \\
\hline $\begin{array}{c}\text { Cabernet Sauvignon, } \\
\text { Feteasca Neagra, } \\
\text { Mamaia, Merlot, } \\
\text { Pinot Noir }\end{array}$ & 2009-2017 & $\begin{array}{l}\text { Phenolics and other chemical compounds in } \\
\text { the } 1600-900 \mathrm{~cm}^{-1} \text { spectral region }\end{array}$ & Different fingerprints & FT-IR & LDA, PCA, PLS-DA & [1] \\
\hline $\begin{array}{l}\text { Cabernet Sauvignon, } \\
\text { Feteasca Neagra, } \\
\text { Mamaia, Merlot, } \\
\text { Pinot Noir }\end{array}$ & 2009-2014 & $\begin{array}{c}\text { Mainly delphinidin-3-O-glucoside, } \\
\text { peonidin-3-O-glucoside, } \\
\text { malvidin-3-O-acetylglucoside, } \\
\text { malvidin-3-O-p-coumarylglucoside, } \\
\text { peonidin-3-O-(6-p-coumaroyl)glucoside }\end{array}$ & $\begin{array}{c}\text { Specific set of } \\
\text { concentrations and ratios of } \\
\text { anthocyanins (expressed in } \\
\text { mg/L of } \\
\text { malvidin-3-O-glucoside) } \\
\text { together with NMR } \\
\text { fingerprint }\end{array}$ & $\begin{array}{l}\text { HPLC-PDA, } \\
\text { NMR }\end{array}$ & LDA, PCA & [58] \\
\hline
\end{tabular}

MD—multidimensional; PLSR—partial least squares regression; SD—standard deviation. 
As discussed before, different classes of phenolic compounds can be combined to get a good set of quality markers. Bellomarino et al. [111] reported that a mix of phenolic acids, flavonoids, and stilbenes was suitable to assess the vintage year of Cabernet Sauvignon wines, since their concentrations changed during aging. The content of tartaric acid, sinapic acid, and (+)-catechin decreased, but the concentrations of vanillic acid and resveratrol increased. Instead, Li et al. [12] applied a set of flavonoids for identifying the vintages of Cabernet Sauvignon that $100 \%$ correctly separated young and old wines and showed $81 \%$ differentiation among young wines. Geana et al. [1] used the FT-IR technique and were focused on the signals in the $1600-900 \mathrm{~cm}^{-1}$ spectral region (phenolic acids, flavonoids, tannins, amino acids, aldehydes, sugars, alcohols, etc.) that were able to separate samples from nine different vintages. Previously, the same research team showed the successful application of the combinations of the NMR's fingerprint, the profile of anthocyanins, and certain anthocyanin ratios (cyanidin-3-O-glucoside/malvidin-3-O-glucoside, petunidin-3-O-glucoside/malvidin-3-O-glucoside, malvidin-3-O-(6-p-coumaroyl)glucoside/malvidin-3-O-glucoside and total anthocyanins/malvidin-3$O$-glucoside) to assess the vintage years from 2009 to 2014 [1].

\section{Conclusions}

Phenolics are important chemical compounds present in wines that can be used as quality and authenticity fingerprints in terms of grape variety, grape species, terroir, winemaking, aging conditions, and vintage. In the last two decades, the research based on the phenolic profiles of wines increased exponentially.

However, the majority of studies concerning quality control were performed on red grape varieties (mainly Cabernet Sauvignon, Merlot, Syrah, Pinot Noir, Tempranillo, and Sangiovese); thus, there are less scientific studies on white wine (mainly Chardonnay, Riesling, and Sauvignon Blanc) and especially on rosé wines. Markers applied for the differentiation according to the origin were phenolic acids for white wines and various flavonoids, especially anthocyanins for red wines. Markers for the vintage year are phenolic acids (white wines) and anthocyanins (red wines, mainly Cabernet Sauvignon). Markers for winemaking and aging in wood tanks are ellagic acid and other phenolic acids, flavonols, and tannins. To differentiate grape varieties, phenolic acids, stilbenes (white wines), and anthocyanins (red wines) were mainly used. Liquid chromatography with various detectors (e.g., diode array detection (DAD) and mass spectrometry (MS)) were the main techniques to determine each chemical marker in wine, in comparison with NMR and vibrational spectroscopy that were mostly used for the geographical origin and vintage evaluation. Concerning statistical analyses, PCA, linear discriminant analysis (LDA), PLS-DA, and analysis of variance (ANOVA) were the most applied. Some publications presented newly observed phenolic compounds that have a high potential to be used as chemical markers in the different fields of quality control. However, wine is a complex food matrix that is influenced by many factors, and not many studies were able to focus on the definition of selected chemical markers able to account for different variables (grape variety, geographical origin, winemaking, aging, and vintage). In fact, it could be argued that this currently very active research field could still present a fragmented landscape. This could be the direct result of the evident dependency of the phenolic profile on many variables at the same time. As a result, few analytical and statistical methods for assessing quality could produce a totally generalized model, but they have had to be contextualized instead for certain factors and within the extent and variety of the dataset investigated. Still, very high rates of correct classifications (based on grape variety, geographical origin, etc.) could be achieved, e.g., by optimizing and refining the applied selection of markers. Many discriminant and classification techniques reported in this review have been applied to wine samples, having phenolic compounds as the principal markers and according to the aim of the model (classification by variety, by origin, etc.). In these examples, when the methods were accordingly calibrated and applied, the performances in classification or discrimination were always very high (in general between $90 \%$ and $100 \%$ ). However, one consequence of the unexplained underlying complexity is that the great part of these studies has produced a set/sets of relationships between only specific phenolic compounds or 
classes, and specific factors, excluding (necessarily) all others; hence, on one side, these models were only validated within the limits of the studies themselves, so representing just a portion of the whole complexity; on the other, their high performance was achieved by limiting the number of factors take into consideration. In addition, often, integrating the results obtained from multiple studies might not be so straightforward, due to the heterogeneity of the methods employed and the requirement for an independent external validation. Indeed, the need for validating the models on external sets of samples is an aspect that requires care. One main limitation to a wider application of the phenolic profiles might also be the need of sufficient sample sizes that are able to sufficiently represent the high number of (known and unknown) study variables at play and their interaction. Finally, a comprehensive view could be achieved only when the full relationships between the phenolic profile and the variables is more deeply understood.

Author Contributions: Conceptualization, V.M., E.L. and E.B.; investigation, V.M.; writing-original draft preparation, V.M. and G.W.; writing-review and editing, V.M., E.L. and E.B.; supervision, E.B.; project administration, E.L. and E.B.; funding acquisition, E.L. and E.B. All authors have read and agreed to the published version of the manuscript.

Funding: This project has been funded by the the WineID Project (TN201A) and c-PAC Project (TN200T) of the Free University of Bozen-Bolzano. This work was supported by the Open Access Publishing Fund of the Free University of Bozen-Bolzano.

Conflicts of Interest: The authors declare no conflict of interests.

\section{References}

1. Geana, E.I.; Ciucure, C.T.; Apetrei, C.; Artem, V. Application of Spectroscopic UV-Vis and FT-IR Screening Techniques Coupled with Multivariate Statistical Analysis for Red Wine Authentication: Varietal and Vintage Year Discrimination. Molecules 2019, 24, 4166. [CrossRef]

2. ICQRF. ICQRF Activity Report; ICQRF: Rome, Italy, 2019; pp. 1-73. Available online: https://www. politicheagricole.it (accessed on 30 September 2020).

3. Reuters. Italian police put cork on 'world' best wine' fakes. World News, 14 October 2020.

4. OLAF. More than $1 \mathrm{~m}$ Litres of Counterfeit Wine and Alcoholic Beverages Seized under OLAF's Lead, Press Release No. 22/2020. 2020. Available online: https://ec.europa.eu/anti-fraud/file/5190/download_en? token=HSWzFi7K (accessed on 30 September 2020).

5. Stój, A.; Kapusta, I.; Domagała, D. Classification of Red Wines Produced from Zweigelt and Rondo Grape Varieties Based on the Analysis of Phenolic Compounds by UPLC-PDA-MS/MS. Molecules 2020, 25, 1342. [CrossRef] [PubMed]

6. Costa, N.L.; Llobodanin, L.A.G.; Castro, I.A.; Barbosa, R. Using Support Vector Machines and neural networks to classify Merlot wines from South America. Inf. Process. Agric. 2019, 6, 265-278. [CrossRef]

7. Anastasiadi, M.; Zira, A.; Magiatis, P.; Haroutounian, S.A.; Skaltsounis, A.L.; Mikros, E. 1H NMR-Based Metabonomics for the Classification of Greek Wines According to Variety, Region, and Vintage. Comparison with HPLC Data. J. Agric. Food Chem. 2009, 57, 11067-11074. [CrossRef] [PubMed]

8. Kupsa, J.; Pavloušek, P.; Kumšta, M.; Lampíř, L. Phenolic profiles of Riesling wines originating from different terroirs of the Czech Republic. Mitt. Klosterneubg. 2017, 67, 182-193.

9. Parpinello, G.P.; Ricci, A.; Arapitsas, P.; Curioni, A.; Moio, L.; Segade, S.R.; Ugliano, M.; Versari, A. Multivariate characterisation of Italian monovarietal red wines using MIR spectroscopy. OENO One 2019, 4, 741-751. [CrossRef]

10. Agatonovic-Kustrin, S.; Milojkovic-Opsenica, D.; Morton, D.W.; Ristivojevic, P. Chemometric characterization of wines according to their HPTLC fingerprints. Eur. Food Res. Technol. 2017, 243, 659-667. [CrossRef]

11. Sartor, S.; Malinovski, L.I.; Caliari, V.; Da Silva, A.L.; Bordignon-Luiz, M.T. Particularities of Syrah wines from different growing regions of Southern Brazil: Grapevine phenology and bioactive compounds. J. Food Sci. Technol. 2017, 54, 1414-1424. [CrossRef]

12. Li, S.; Zhu, B.; Reeves, M.J.; Duan, C. Phenolic Analysis and Theoretic Design for Chinese Commercial Wines' Authentication. J. Food Sci. 2018, 83, 30-39. [CrossRef] 
13. Pavloušek, P.; Kumšta, M. Authentication of Riesling Wines from the Czech Republic on the Basis of the Non-flavonoid Phenolic Compounds. Czech J. Food Sci. 2013, 31, 474-482. [CrossRef]

14. Pisano, P.L.; Silva, M.F.; Olivieri, A.C. Anthocyanins as markers for the classification of Argentinean wines according to botanical and geographical origin. Chemometric modeling of liquid chromatography-mass spectrometry data. Food Chem. 2015, 175, 174-180. [CrossRef] [PubMed]

15. Urvieta, R.; Buscema, F.; Bottini, R.; Coste, B.; Fontana, A. Phenolic and sensory profiles discriminate geographical indications for Malbec wines from different regions of Mendoza, Argentina. Food Chem. 2018, 265, 120-127. [CrossRef] [PubMed]

16. OIV. International Standard for the Labelling of Wines; Organisation Internationale de la Vigne et du Vin: Paris, France, 2015; pp. 1-15. Available online: http://www.oiv.int/public/medias/4776/oiv-wine-labelling-standarden-2015.pdf (accessed on 30 September 2020).

17. Chira, K.; Pacella, N.; Jourdes, M.; Teissedre, P.L. Chemical and sensory evaluation of Bordeaux wines (Cabernet-Sauvignon and Merlot) and correlation with wine age. Food Chem. 2011, 126, 1971-1977. [CrossRef] [PubMed]

18. Heras-Roger, J.; Díaz-Romero, C.; Darias-Martín, J. A comprehensive study of red wine properties according to variety. Food Chem. 2016, 196, 1224-1231. [CrossRef] [PubMed]

19. Lukic, I.; Radek, S.; Budic-Leton, I.; Bubola, M.; Vrhovsek, U. Targeted UPLC-QqQ-MS/MS profiling of phenolic compounds for differentiation of monovarietal wines and corroboration of particular varietal typicity concepts. Food Chem. 2019, 300, 125251. [CrossRef] [PubMed]

20. Proestos, C.; Tzachristas, A. Polyphenols in Wine Authenticity. In Scholarly Community Encyclopedia; MDPI: Basel, Switzerland, 2020.

21. Kalogiouri, N.P.; Samanidou, V.F. Liquid chromatographic methods coupled to chemometrics: A short review to present the key workflow for the investigation of wine phenolic composition as it is affected by environmental factors. Environ. Sci. Pollut. Res. 2020, 1-15. [CrossRef]

22. Cejudo-Bastante, M.J.; Vicario, A.; Guillén, D.A.; Hermosín-Gutiérrez, I.; Pérez-Coello, M.S. Phenolic characterization of minor red grape varieties grown in Castilla-La Mancha region in different vinification stages. Eur. Food Res. Technol. 2015, 240, 595-607. [CrossRef]

23. Ragusa, A.; Centonze, C.; Grasso, M.E.; Latronico, M.F.; Mastrangelo, P.F.; Sparascio, F.; Fanizzi, F.P.; Maffia, M. A Comparative Study of Phenols in Apulian Italian Wines. Foods 2017, 6, 24. [CrossRef]

24. Di Lecce, G.; Boselli, E.; D’Ignazi, G.; Frega, N.G. Evolution of phenolics and glutathione in Verdicchio wine obtained with maceration under reductive conditions. LWT 2013, 53, 54-60. [CrossRef]

25. Boselli, E.; Di Lecce, G.; Alberti, F.; Frega, N.G. Nitrogen gas affects the quality and the phenolic profile of must obtained from vacuum-pressed white grapes. LWT 2010, 43, 1494-1500. [CrossRef]

26. Berente, B.; De la Calle García, D.; Reichenbächer, M.; Danzer, K. Method development for the determination of anthocyanins in red wines by high-performance liquid chromatography and classification of German red wines by means of multivariate statistical methods. J. Chromatogr. A 2000, 871, 95-103. [CrossRef]

27. Revilla, E.; Garcia-Beneytez, E.; Cabello, F.; Martin-Ortega, G.; Ryan, J. Value of high-performance liquid chromatographic analysis of anthocyanins in the differentiation of red grape cultivars and red wines made from them. J. Chromatogr. A 2001, 915, 53-60. [CrossRef]

28. Castillo-Munoz, N.; Gomez-Alonso, S.; Garcia-Romero, E.; Hermosian-Gutierrez, I. Flavonol Profiles of Vitis vinifera Red Grapes and Their Single-Cultivar Wines. J. Agric. Food Chem. 2007, 55, 992-1002. [CrossRef] [PubMed]

29. Vergara, C.; Von Baer, D.; Mardones, C.; Gutierrez, L.; Hermosin-Gutierrez, I.; Castillo-Munoz, N. Flavonol profiles for varietal differentiation between Carmenere and Merlot wines produced in Chile: HPLC and chemometric analysis. J. Chil. Chem. Soc. 2011, 56, 827-832. [CrossRef]

30. Mattivi, F.; Vrhovsek, U.; Masuero, D.; Trainotti, D. Differences in the amount and structure of extractable skin and seed tannins amongst red grape varieties. Aust. J. Grape Wine Res. 2008, 15, 27-35. [CrossRef]

31. Chira, K.; Jourdes, M.; Teissedre, P. Cabernet sauvignon red wine astringency quality control by tannin characterization and polymerization during storage. Eur. Food Res. Technol. 2012, 234, 253-261. [CrossRef]

32. Basalekou, M.; Kallithraka, S.; Tarantilis, P.A.; Kotseridis, Y.; Pappas, C. Ellagitannins in wines: Future prospects in methods of analysis using FT-IR spectroscopy. LWT 2019, 101, 48-53. [CrossRef] 
33. Motta, S.; Guaita, M.; Cassino, C.; Bosso, A. Relationship between polyphenolic content, antioxidant properties and oxygen consumption rate of different tannins in a model wine solution. Food Chem. 2020, 313, 126045. [CrossRef]

34. Sun, B.; Leandro, C.; da Silva, J.M.R.; Spranger, I. Separation of Grape and Wine Proanthocyanidins According to Their Degree of Polymerization. J. Agric. Food Chem. 1998, 46, 1390-1396. [CrossRef]

35. Llaudy, M.; Canals, R.; Canals, J.M.; Rozéz, N.; Arola, L.; Zamora, F. New method for evaluating astringency in red wine. J. Agric. Food Chem. 2004, 52, 742-746. [CrossRef]

36. Mazza, G. Anthocyanins in grapes and grapes products. Crit. Rev. Food Sci. Nutr. 1995, 35, 341-371. [CrossRef] [PubMed]

37. Baron, M.; Sochor, J.; Tomaskova, L.; Prusova, B.; Kumsta, M. Study on Antioxidant Components in Rosé Wine Originating from the Wine Growing Region of Moravia, Czech Republic. Erwerbs-Obstbau 2017, 59, 253-262. [CrossRef]

38. Martelo-Vidal, M.J.; Vázquez, M. Determination of polyphenolic compounds of red wines by UV-VIS-NIR spectroscopy and chemometrics tools. Food Chem. 2014, 158, 28-34. [CrossRef] [PubMed]

39. Ragusa, A.; Centonze, C.; Grasso, M.E.; Latronico, M.F.; Mastrangelo, P.F.; Sparascio, F.; Maffia, M. HPLC Analysis of Phenols in Negroamaro and Primitivo Red Wines from Salento. Foods 2019, 8, 45. [CrossRef]

40. Lampir, L.; Pavlousek, P. Influence of Locality on Content of Phenolic Compounds in White Wines. Czech J. Food Sci. 2013, 31, 619-626. [CrossRef]

41. Hornedo-Ortega, R.; González-Centeno, M.R.; Chira, K.; Jourdes, M.; Teissedre, P.L. Phenolic Compounds of Grapes and Wines: Key Compounds and Implications in Sensory Perception. In Winemaking-Stabilization, Aging Chemistry and Biochemistry; IntechOpen: Rijeka, Croatia, 2020.

42. Lampir, L. Varietal Differentiation of White Wines on the Basis of Phenolic Compounds profile. Czech J. Food Sci. 2013, 31, 172-179. [CrossRef]

43. Magdas, D.A.; Pirnau, A.; Feher, I.; Guyon, F.; Cozar, B.I. Alternative approach of applying $1 \mathrm{H}$ NMR in conjunction with chemometrics for wine classification. LWT 2019, 109, 422-428. [CrossRef]

44. Ali, K.; Maltese, F.; Toepfer, R.; Choi, Y.H.; Verpoorte, R. Metabolic characterization of Palatinate German white wines according to sensory attributes, varieties, and vintages using NMR spectroscopy and multivariate data analyses. J. Biomol. NMR 2011, 49, 255-266. [CrossRef]

45. Godelmann, R.; Fang, F.; Humpfer, E.; Schütz, B.; Bansbach, M.; Schäfer, H.; Spraul, M. Targeted and Nontargeted Wine Analysis by $1 \mathrm{H}$ NMR Spectroscopy Combined with Multivariate Statistical Analysis. Differentiation of Important Parameters: Grape Variety, Geographical Origin, Year of Vintage. J. Agric. Food Chem. 2013, 61, 5610-5619. [CrossRef]

46. Boselli, E.; Minardi, M.; Giomo, A.; Frega, N.G. Phenolic composition and quality of white d.o.c. wines from Marche (Italy). Anal. Chim. Acta 2006, 563, 93-100. [CrossRef]

47. La Notte, E.; Liuzzi, V.A.; Esti, M. I componenti polifenolici del vino-Nota II: Gli antociani in relazione a differenti sistemi di vinificazione. Vigne Vini 1992, 10, 49-55.

48. Storchi, P.; Baldi, M.; Faviere, V.; Giannetti, F.; Leprini, M.; Peduto, F. Valutazione del comportamento produttivo e della componente fenolica in vitigni a bacca nera coltivati in Toscana. Quaderni di Scienze Viticole ed Enologiche 2008, 30, 3-21.

49. Wenzel, K.; Dittrich, H.H.; Heimfarth, M. Die Zusammensetzung der Anthocyane in den Beeren verschiedener Rebsorten. Vitis 1987, 26, 65-78.

50. Gonzalez-Neves, G.; Favre, G.; Piccardo, D.; Gil, G. Anthocyanin profile of young red wines of Tannat, Syrah and Merlot made using maceration enzymes and cold soak. Int. J. Food Sci. 2016, 51, 260-267. [CrossRef]

51. García-Beneytez, E.; Cabello, F.; Revilla, E. Analysis of Grape and Wine Anthocyanins by HPLC-MS. J. Agric. Food Chem. 2003, 51, 5622-5629. [CrossRef]

52. Muccillo, L.; Gambuti, A.; Frusciante, L.; Iorizzo, M.; Moio, L.; Raieta, K.; Rinaldi, A.; Colantuoni, V.; Aversano, R. Biochemical features of native red wines and genetic diversity of the corresponding grape varieties from Campania region. Food Chem. 2014, 143, 506-513. [CrossRef]

53. Kumšta, M.; Pavloušek, P.; Kárník, P. Use of Anthocyanin Profiles When Differentiating Individual Varietal Wines and Terroirs. Food Technol. Biotechnol. 2014, 52, 383-390. [CrossRef] 
54. Ferrandino, A.; Guidoni, S.; Mannini, F. Grape Quality Parameters and Polyphenolic Content of Different 'Barbera' and 'Nebbiolo' (Vitis vinifera L.) Clones as Influenced by Environmental Conditions-Preliminary Results. Acta Hortic. 2007, 754, 437-442. [CrossRef]

55. Río Segade, S.; Pace, C.; Torchio, F.; Giacosa, S.; Gerbi, V.; Rolle, L. Impact of maceration enzymes on skin softening and relationship with anthocyanin extraction in wine grapes with different anthocyanin profiles. Food Res. Int. 2015, 71, 50-57. [CrossRef]

56. Giacosa, S.; Ossola, C.; Botto, R.; Río Segade, S.; Paissoni, M.A.; Pollon, M.; Gerbi, V.; Rolle, L. Impact of specific inactive dry yeast application on grape skin mechanical properties, phenolic compounds extractability, and wine composition. Food Res. Int. 2019, 116, 1084-1093. [CrossRef]

57. Arapitsas, P.; Ugliano, M.; Marangon, M.; Piombino, P.; Rolle, L.; Gerbi, G.; Versari, A.; Mattivi, F. Use of Untargeted Liquid Chromatography-Mass Spectrometry Metabolome To Discriminate Italian Monovarietal Red Wines, Produced in Their Different Terroirs. J. Agric. Food Chem. 2020. [CrossRef] [PubMed]

58. Geana, E.I.; Popescu, R.; Costinel, D.; Dinca, O.R.; Ionete, R.E.; Stefanescu, I.; Artem, V.; Bala, C. Classification of red wines using suitable markers coupled with multivariate statistic analysis. Food Chem. 2016, 192, 1015-1024. [CrossRef] [PubMed]

59. Mayr, C.M.; De Rosso, M.; Dalla Vedova, A.; Flamini, R. High-Resolution Mass Spectrometry Identification of Secondary Metabolites in Four Red Grape Varieties Potentially Useful as Traceability Markers of Wines. Beverages 2018, 4, 74. [CrossRef]

60. De Rosso, M.; Mayr, C.M.; Girardi, G.; Dalla Vedova, A.; Flamini, R. High-resolution mass spectrometry metabolomics of grape chemical markers to reveal use of not-allowed varieties in the production of Amarone and Recioto wines. Metabolomics 2018, 14, 124. [CrossRef]

61. Pisano, P.L.; Silva, M.F.; Olivieri, A.C. Exploration of liquid chromatographic-diode array data for Argentinean wines by extended multivariate curve resolution. Chemometr. Intell. Lab. 2014, 132, 1-7. [CrossRef]

62. Fanzone, M.; Zamora, F.; Jofre, V.; Assof, M.; Gomez-Cordovesc, C.; Pena-Neirad, A. Phenolic characterisation of red wine from different grape varieties cultivated in Mendoza province (Argentina). J. Sci. Food Agric. 2012, 92, 704-718. [CrossRef]

63. Monagas, M.; Gomez-Cordoves, C.; Bartolome, B.; Laureano, O.; da Silva, J.R.M. Monomeric, Oligomeric, and Polymeric Flavan-3-ol Composition of Wines and Grapes from Vitis vinifera L. Cv. Graciano, Tempranillo, and Cabernet Sauvignon. J. Agric. Food Chem. 2003, 51, 6475-6481. [CrossRef]

64. Minnaar, P.P.; Booyse, M. Differentiation among Young and Market-ready Cabernet Sauvignon, Pinotage and Shiraz Wines: Application of Canonical Discriminant Analysis using Flavonoid and Non-Flavonoid Compositional Data. S. Afr. J. Enol. Vitic. 2011, 32, 251-261. [CrossRef]

65. Gris, E.F.; Mattivi, F.; Ferreira, E.A.; Vrhovsek, U.; Pedrosa, R.C.; Bordignon-Luiz, M.T. Proanthocyanidin profile and antioxidant capacity of Brazilian Vitis vinifera red wines. Food Chem. 2011, 126, 213-220. [CrossRef]

66. Sun, X.; Li, L.; Ma, T.; Liu, X.; Huang, W.; Zhan, J. Profiles of Phenolic Acids and Flavan-3-ols for Select Chinese Red Wines: A Comparison and Differentiation According to Geographic Origin and Grape Variety. J. Food Sci. 2015, 80, C2170-C2179. [CrossRef]

67. Pajović-Šćepanović, R.; Wendelin, S.; Eder, R. Phenolic composition and varietal discrimination of Montenegrin red wines (Vitis vinifera var. Vranac, Kratošija, and Cabernet Sauvignon). Eur. Food Res. Technol. 2018, 244, 2243-2254. [CrossRef]

68. Salvatore, E.; Cocchi, M.; Marchetti, A.; Marini, F.; De Juan, A. Determination of phenolic compounds and authentication of PDO Lambrusco wines by HPLC-DAD and chemometric techniques. Anal. Chim. Acta 2013, 761, 34-45. [CrossRef] [PubMed]

69. Magdas, D.A.; Pinzaru, S.C.; Guyon, F.; Feher, I.; Cozar, B.I. Application of SERS technique in white wines discrimination. Food Control 2018, 92, 30-36. [CrossRef]

70. Magdas, D.A.; Guyon, F.; Feher, I.; Pinzaru, S.C. Wine discrimination based on chemometric analysis of untargeted markers using FT-Raman spectroscopy. Food Control 2018, 85, 385-391. [CrossRef]

71. Magdas, D.A.; Cozar, B.I.; Feher, I.; Guyon, F.; Dehelean, A.; Pinzaru, S.C. Testing the limits of FT-Raman spectroscopy for wine authentication: Cultivar, geographical origin, vintage and terroir effect influence. Sci. Rep. 2019, 27, 19954. [CrossRef] [PubMed]

72. Longo, E.; Merkytė, V.; Rossetti, F.; Teissedre, P.L.; Jourdes, M.; Boselli, E. Relative abundances of novel cyclic prodelphinidins in wine depending on the grape variety. Mass Spectrom. 2018, 53, 1116-1125. [CrossRef] 
73. Longo, E.; Rossetti, F.; Jouin, A.; Teissedre, P.; Jourdes, M.; Boselli, E. Distribution of crown hexameric procyanidin and its tetrameric and pentameric congeners in red and white wines. Food Chem. 2019, 299, 125125. [CrossRef]

74. Zeng, L.; Pons-Mercadé, P.; Richard, T.; Krisa, S.; Teissedre, P.L.; Jourdes, M. Crown procyanidin tetramer: A procyanidin with an unusual cyclic skeleton with a potent protective effect against amyloid- $\beta$-induced toxicity. Molecules 2019, 24, 1915. [CrossRef]

75. Favre, G.; González-Neves, G.; Piccardo, D.; Gómez-Alonso, S.; Pérez-Navarro, J.; Hermosín-Gutiérrez, I. New acylated flavonols identified in Vitis vinifera grapes and wines. Food Res. Int. 2018, 112, 98-107. [CrossRef]

76. Baldi, A.; Romani, A.; Mulinacci, N.; Vincieri, F.F.; Casetta, B. HPLC/MS Application to Anthocyanins of Vitis vinifera L. J. Agric. Food Chem. 1995, 43, 2104-2109. [CrossRef]

77. Mattivi, F.; Cova, G.; Dalla Serra, A.; Soligo, S. Recupero, Conservazione e Valorizzazione del Germoplasma Viticolo Veneto; Veneto Agricoltura: Legnaro, Italy, 2004; pp. 17-19.

78. Boselli, E.; Giomo, A.; Minardi, M.; Frega, N.G. Characterization of phenolics in Lacrima di Morro d'Alba wine and role on its sensory attributes. Eur. Food Res. Technol. 2008, 227, 709-720. [CrossRef]

79. Xing, R.R.; Li, S.Y.; He, F.; Yang, Z.; Duan, C.Q.; Li, Z.; Wang, J.; Pan, Q.H. Mass spectrometric and enzymatic evidence confirm the existence of anthocyanidin 3,5-O-diglucosides in cabernet sauvignon (Vitis vinifera L.) grape berries. J. Agric. Food Chem. 2015, 63, 3251-3260. [CrossRef] [PubMed]

80. OIV. Maximum Acceptable Limits of Various Substances Contained in Wine; OENO, OIV-MA-C1-01; OIV: Paris, France, 2015; pp. 1-4. Available online: http://www.oiv.int/public/medias/3741/e-code-annex-maximumacceptable-limits.pdf (accessed on 30 September 2020).

81. Nixdorf, S.L.; Hermosín-Gutiérrez, I. Brazilian red wines made from the hybrid grape cultivar Isabel: Phenolic composition and antioxidant capacity. Anal. Chim. Acta 2010, 659, 208-215. [CrossRef] [PubMed]

82. Meng, J.; Xu, T.; Qin, M.; Zhuang, X.; Fang, Y.; Zhang, Z. Phenolic characterization of young wines made from spine grape (Vitis davidii Foex) grown in Chongyi County (China). Food Res. Int. 2012, 49, 664-671. [CrossRef]

83. Li, S.; He, F.; Zhu, B.; Wang, J.; Duan, C. Comparison of phenolic and chromatic characteristics of dry red wines made from native Chinese grape species and Vitis vinifera. Int. J. Food Prop. 2017, 20, 2134-2146. [CrossRef]

84. Burns, J.; Mullen, W.; Landrault, N.; Teissedre, P.L.; Lean, M.E.J.; Crozier, A. Variations in the Profile and Content of Anthocyanins in Wines Made from Cabernet Sauvignon and Hybrid Grapes. J. Agric. Food Chem. 2002, 50, 4096-4102. [CrossRef]

85. Gougeon, L.; Da Costa, G.; Le Mao, I.; Ma, W.; Teissedre, P.L.; Guyon, F.; Richard, T. Wine Analysis and Authenticity Using 1H-NMR Metabolomics Data: Application to Chinese Wines. Food Anal. Methods 2018, 11, 3425-3434. [CrossRef]

86. Giaccio, M.; Vicentini, A. Determination of the geographical origin of wines by means of the mineral content and the stable isotope ratios: A review. J. Commod. Sci. Technol. Qual. 2008, 47, 267-284.

87. Kumšta, M.; Pavloušek, P.; Kupsa, J. Phenolic Profile in Czech White Wines from Different Terroirs. Food Sci. Biotechnol. 2012, 21, 1593-1601. [CrossRef]

88. Rocchetti, G.; Gatti, M.; Bavaresco, L.; Lucini, L. Untargeted metabolomics to investigate the phenolic composition of Chardonnay wines from different origins. J. Food Compost. Anal. 2018, 71, 87-93. [CrossRef]

89. Bellomarino, S.A.; Conlan, X.A.; Parker, R.M.; Barnett, N.W.; Adams, M.J. Geographical classification of some Australian wines by discriminant analysis using HPLC with UV and chemiluminescence detection. Talanta 2009, 80, 833-838. [CrossRef] [PubMed]

90. Lambert, M.; Meudec, E.; Verbaere, A.; Mazerolles, G.; Wirth, J.; Masson, G.; Cheynier, V.; Sommerer, N. A High-Throughput UHPLC-QqQ-MS Method for Polyphenol Profiling in Rosé Wines. Molecules 2015, 20, 7890-7914. [CrossRef] [PubMed]

91. Radovanovic, B.C.; Radovanovic, A.N.; Souquet, J. Phenolic profile and free radical-scavenging activity of Cabernet Sauvignon wines of different geographical origins from the Balkan region. J. Sci. Food Agric. 2010, 90, 2455-2461. [CrossRef] [PubMed]

92. Jiang, B.; Zhang, Z.W. Free Radical-scavenging Activity and Anthocyanin Profiles of Cabernet Sauvignon and Merlot Wines from Four Wine Grapegrowing Regions in China. S. Afr. J. Enol. Vitic. 2019, 40, 1-10. [CrossRef] 
93. Radovanovic, B.; Radovanovic, A. Free Radical Scavenging Activity and Anthocyanin Profile of Cabernet Sauvignon Wines from the Balkan Region. Molecules 2010, 15, 4213-4226. [CrossRef]

94. Acevedo, F.J.; Jimenez, J.; Maldonado, S.; Dominguez, E.; Narvaez, A. Classification of Wines Produced in Specific Regions by UV-Visible Spectroscopy Combined with Support Vector Machines. J. Agric. Food Chem. 2007, 55, 6842-6849. [CrossRef] [PubMed]

95. Sacchi, K.L.; Bisson, L.F.; Adams, D.O. A Review of the Effect of Winemaking Techniques on Phenolic Extraction in Red Wines. Am. J. Enol. Vitic. 2005, 56, 197-206.

96. Bosch-Fusté, J.; Sartini, E.; Flores-Rubio, C.; Caixach, J.; López-Tamames, E.; Buxaderas, S. Viability of total phenol index value as quality marker of sparkling wines, "cavas". Food Chem. 2009, 114, 782-790. [CrossRef]

97. Izquierdo-Llopart, A.; Saurina, J. Characterization of Sparkling Wines According to Polyphenolic Profiles Obtained by HPLC-UV/Vis and Principal Component Analysis. Foods 2019, 8, 22. [CrossRef]

98. Dupas de Matos, A.; Longo, E.; Chiotti, D.; Pedri, U.; Eisenstecken, D.; Sanoll, C.; Robatscher, P.; Boselli, E. Pinot Blanc: Impact of the Winemaking Variables on the Evolution of the Phenolic, Volatile and Sensory Profiles. Foods 2020, 9, 499. [CrossRef]

99. Suriano, S.; Alba, V.; Tarricone, L.; Di Gennaro, D. Maceration with stems contact fermentation: Effect on proanthocyanidins compounds and color in Primitivo red wines. Food Chem. 2015, 177, 382-389. [CrossRef] [PubMed]

100. Merkytè, V.; Dupas de Matos, A.; Longo, E.; Boselli, E. Cyclic proanthocyanidins in Pinot Noir wine. Ital. J. Food Sci. 2020, 32, 337-351.

101. Lorenzo, C.; Pardo, F.; Zalacain, A.; Alonso, G.L.; Salinas, M.R. Effect of Red Grapes Co-winemaking in Polyphenols and Colour of Wines. J. Agric. Food Chem. 2005, 53, 7609-7616. [CrossRef] [PubMed]

102. Loizzo, M.R.; Bonesi, M.; Di Lecce, G.; Boselli, E.; Tundis, R.; Pugliesen, A.; Menichini, F.; Frega, N.G. Phenolics, Aroma Profile, and In Vitro Antioxidant Activity of Italian Dessert Passito Wine from Saracena (Italy). J. Food Sci. 2013, 78, C704-C708. [CrossRef]

103. Matejicek, D.; Mikes, O.; Klejdus, B.; Sterbova, D.; Kuban, V. Changes in contents of phenolic compounds during maturing of barrique red wines. Food Chem. 2005, 90, 791-800. [CrossRef]

104. Arapitsas, P.; Guella, G.; Mattivi, F. The impact of $\mathrm{SO}_{2}$ on wine flavanols and indoles in relation to wine style and age. Sci. Rep. 2018, 8, 858-870. [CrossRef]

105. Sanz, M.; Fernández de Simón, B.; Esteruelas, E.; Munoza, A.M.; Cadahía, E.; Hernández, M.T.; Estrella, I.; Martinez, J. Polyphenols in red wine aged in acacia (Robinia pseudoacacia) and oak (Quercus petraea) wood barrels. Anal. Chim. Acta 2012, 732, 83-90. [CrossRef]

106. Alañón, M.E.; Schumacher, R.; Castro-Vázquez, L.; Díaz-Maroto, M.C.; Hermosín-Gutiérrez, I.; Pérez-Coello, M.S. Enological potential of chestnut wood for aging Tempranillo wines Part II: Phenolic compounds and chromatic characteristics. Food. Res. Int. 2013, 51, 536-543. [CrossRef]

107. Chinnici, F.; Natali, N.; Bellachioma, A.; Versari, A.; Riponi, C. Changes in phenolic composition of red wines aged in cherry wood. LWT 2015, 60,977-984. [CrossRef]

108. Del Alamo, M.; Nevares, I.; Gallego, L.; Martina, C.; Merino, S. Aging markers from bottled red wine aged with chips, staves and barrels. Anal. Chim. Acta 2008, 621, 86-99. [CrossRef]

109. Ortega-Heras, M.; Pérez-Magariño, S.; Cano-Mozo, E.; González-San José, M.L. Differences in the phenolic composition and sensory profile between red wines aged in oak barrels and wines aged with oak chips. LWT 2010, 43, 1533-1541. [CrossRef]

110. Baiano, A.; De Gianni, A.; Mentana, A.; Quinto, M.; Centonze, D.; Del Nobile, M.A. Effects of the treatment with oak chips on color-related phenolics, volatile composition, and sensory profile of red wines: The case of Aglianico and Montepulciano. Eur. Food. Res. Technol. 2016, 242, 745-767. [CrossRef]

111. Basalekou, M.; Pappas, C.; Tarantilis, P.; Kotseridis, Y.; Kallithraka, S. Wine authentication with Fourier Transform Infrared Spectroscopy: A feasibility study on variety, type of barrel wood and ageing time classification. Int. J. Food Sci. 2017, 52, 1307-1313. [CrossRef]

112. Bellomarino, S.A.; Parker, R.M.; Conlan, X.A.; Barnett, N.W.; Adams, M.J. Partial least squares and principal components analysis of wine vintage by high performance liquid chromatography with chemiluminescence detection. Anal. Chim. Acta 2010, 678, 34-38. [CrossRef]

113. Schwarz, M.; Hofmann, G.; Winterhalter, P. Investigations on Anthocyanins in Wines from Vitis vinifera cv. Pinotage: Factors Influencing the Formation of Pinotin A and Its Correlation with Wine Age. J. Agric. Food Chem. 2004, 52, 498-504. [CrossRef] 
114. Revilla, E.; Garcia-Beneytez, E.; Cabello, F. Anthocyanin fingerprint of clones of Tempranillo grapes and wines made with them. Aust. J. Grape Wine Res. 2009, 15, 70-78. [CrossRef]

115. Arapitsas, P.; Perenzoni, D.; Nicolini, G.; Mattivi, F. Study of Sangiovese Wines Pigment Profile by UHPLC-MS/MS. J. Agric. Food Chem. 2012, 60, 10461-10471. [CrossRef]

116. Eder, R.; Beyer, B.; Patzl-Fischerleitner, E.; Wendelin, S.; Hann, S. Determination of pyranoanthocyanine and malvidin-3-glucoside content in red wine of different vintages via LC-MS/ESI. Mitt. Klosterneubg. 2014, 64, 183-192.

Publisher's Note: MDPI stays neutral with regard to jurisdictional claims in published maps and institutional affiliations.

(C) 2020 by the authors. Licensee MDPI, Basel, Switzerland. This article is an open access article distributed under the terms and conditions of the Creative Commons Attribution (CC BY) license (http://creativecommons.org/licenses/by/4.0/). 